\title{
Potential Muscle Biomarkers of Chronic Myalgia in Humans - A Systematic Review of Microdialysis Studies
}

\author{
Björn Gerdle and Britt Larsson \\ Rehabilitation Medicine, Faculty of Health Sciences, Linköping University, \\ Linköping, and Pain and Rehabilitation Centre, University Hospital, Linköping, \\ Sweden
}

\section{Introduction}

\subsection{Epidemiology}

Approximately $20 \%$ of the European population report severe chronic pain (Breivik et al., 2006), with higher prevalences in women and in lower income groups (Gerdle et al., 2004; Larsson et al., 2007). Common chronic pain conditions are localized neck-shoulder pain including trapezius myalgia (prevalence in population 10-20\%) (Lidgren, 2008), chronic whiplash associated disorders (WAD) (prevalence in the population 1.5\%) (Guez et al., 2002), and chronic widespread pain (CWSP) (prevalence in population 5-10\%) (Gerdle et al., 2008a). Chronic pain is associated with disability, low quality of life, and substantial socioeconomic costs (Breivik et al., 2006; Phillips, 2006; SBU, 2006).

\subsection{Development of chronic myalgia - Chronic trapezius myalgia as an example}

There is a connection between physical demands, psychosocial demands, and the risk of persistent muscle pain (Bernard, 1997; Punnett \& Wegman, 2004); however, the mechanisms behind chronic myalgia are poorly understood. Myalgia usually starts with a feeling of tiredness and stiffness. At the beginning, the initial intermittent stage, pain can be alleviated for short or long periods. Chronic regional myalgia (CRM) in the neck-shoulder area often gradually becomes more easily triggered and more diffuse and can be spread to include most of the body (CWSP). CWSP includes fibromyalgia, a subgroup characterized by widespread hyperalgesia. The risk factors for the transition from a local/regional pain condition to CWSP are poorly understood (Larsson et al., in press). The diagnoses CRM (e.g., chronic trapezius myalgia) and CWSP are settled by careful anamnesis and clinical examinations that reveal tender muscle at palpation corresponding to the reported painful areas.

\subsection{Neurobiological alterations in chronic pain}

Acute pain results from a complex integrated series of events at peripheral and central levels. In healthy subjects, mechanisms related to acute pain might not necessarily be valid in subjects with subchronic, intermittent, or chronic pain. 
Pace et al. suggested two types of persistent chronic pain: 1) nociceptive/inflammatory pain and 2) neuropathic pain (Pace et al., 2006). The present study mainly discusses nociceptive/inflammatory pain. Chronic pain is more complex than acute pain as extensive short-term and long-term plastic and sometimes permanent changes (including peripheral and/or central hyperexcitability/sensitization) of the pain transmission system can occur at different levels (Kuner, 2010; Reichling \& Levine, 2009) and by the modification of psychological (e.g., attentional, emotional, and anticipation status) and context factors (Grachev et al., 2000; Hunt \& Mantyh, 2002; Petersen-Felix \& Curatolo, 2002; Schmidt-Wilcke, 2008; Wilder-Smith et al., 2002; Woolf \& Salter, 2000). Different structures in the brain vaguely labelled as the pain matrix (Iannetti \& Mouraux, 2010; Lee \& Tracey, 2010; Legrain et al., 2011) - are dynamically involved in processing of nociception and pain (including emotions, cognitions, and motivation) (Ossipov et al., 2010). In patients with chronic pain conditions, a pain matrix shows different types of alterations including morphological changes (Apkarian, 2008; Schweinhardt \& Bushnell, 2010), indicating that different chronic pain conditions exhibit unique anatomical "brain signatures" (Baliki et al., 2011).

Descending supraspinal control of spinal nociception originates from many brain regions (Heinricher et al., 2009; Ossipov et al., 2010). The descending supraspinal control includes a dynamic balance between inhibiting and facilitating mechanisms that can be altered due to behavioural, emotional, and pathological states (Heinricher et al., 2009; Ossipov et al., 2010). When the system shifts towards inhibition, hyposensibility or lack of pain in spite of inputs from peripheral tissue may result (Heinricher et al., 2009; Kuner, 2010; Porreca et al., 2002; Ren \& Dubner, 2002; Robinson \& Zhuo, 2002; Wilder-Smith et al., 2002). The evolutionary value of this is that the organism can ignore pain during critical situations, e.g., flight or fight scenarios (Kuner, 2010). A facilitating shift of the descending system has been reported for different groups of patients with persistent pain (Heinricher et al., 2009; Julien et al., 2005; Kuner, 2010; Porreca et al., 2002; Ren \& Dubner, 2002; Robinson \& Zhuo, 2002; WilderSmith et al., 2002).

Decreased production of substances such as endorphins or gamma-aminobutyric acid (GABA), the most common inhibitory neurotransmitter in the central nervous system, may contribute to disturbances in pain inhibition. Animal and human studies have confirmed that the endogenous opioid system and GABA play a role in the modulation of pain. However, the understanding of the mechanisms, including the peripheral balances between nociceptive and anitinociceptive processes, behind chronic myalgia is incomplete. The analgesic properties of exogenous cannabinoids have been recognized for many years. Data clearly implicate endocannabinoids as endogenous tonic pain controlling molecules (Agarwal et al., 2007; Richardson et al., 1998; Walker \& Huang, 2002), but little is known as to whether peripheral endocannabinoid signalling is disturbed in human pain.

\subsection{Muscle nociception and peripheral sensitization}

Neurophysiological studies have indicated that small-diameter, slowly conducting afferent nerve fibres from skeletal muscle -free nerve endings of group III (Aס) and IV afferent (C) fibres - have to be excited to elicit pain (Mense, 2003). The nociceptor is specialized to respond to noxious stimuli and communicate this information to the CNS. Nociceptors or noxious stimulus detectors (Woolf \& Ma, 2007) are sensitive to chemical substances released from damaged or overloaded cells and excessive tissue deformation (Coutaux et al., 2005; Mense, 1993). Nociceptors respond to single or combinations of stimuli: noxious mechanical 
stimuli, temperature, and chemical substances such as serotonin $\mathrm{H}^{+},(5-\mathrm{HT})$, bradykinin $(\mathrm{BKN})$, glutamate, prostaglandin $\mathrm{E}_{2}\left(\mathrm{PGE}_{2}\right)$, substance $\mathrm{P}$, nerve growth factor (NGF), ATP, and potassium (Coutaux et al., 2005; Mense, 1993; 2009). Administration of any of these substances, alone or in combination, results in excitation of nociceptors (Mense, 1993). The relative effectiveness of these substances is unknown. In a pathophysiological situation due to trauma or inflammation, a combination of substances acts on the nociceptors (the inflammatory "soup" or "cocktail")(Mense, 2009). The nociceptor is not a static detector as plastic changes can occur such as peripheral sensitization (Woolf \& Ma, 2007). A sensitized nociceptor has a lowered threshold for activation and can thus be activated by stimuli that are normally innocuous (Coutaux et al., 2005; Mense, 1993). Several substances - e.g., $\mathrm{H}^{+}$, $\mathrm{NO}, \mathrm{K}^{+}, \mathrm{ATP}$, bradykinin (BKN), PGE 2 NGF, TNF-a, IL-6, and glutamate - are known to cause peripheral sensitization (Coutaux et al., 2005; Mense, 2009; Momin \& McNaughton, 2009). The action of these substances is mediated by their specific receptors mainly found in three classes: 1) G protein coupled receptors; 2) receptor tyrosine kinases; and 3) ionotropic receptors/ion channels (Linley et al., 2010). Sensitization is often accompanied by an increase in the sensitive area (Mense, 1993). In addition, other alterations of the nociceptors, including activation of silent nociceptors, have been found as the result of injury or inflammation (Schaible et al., 2009). When persistent alterations in the nociceptors as the result of induced gene transcription and protein synthesis drive pain in the absence of noxious stimuli these alterations represent a pathological condition (Woolf \& Ma, 2007).

\subsection{The bio-psycho-social model of chronic pain}

The net result of the above mentioned and other alterations are clinically registered as pain hypersensitivity - an increased responsiveness to nociception and sometimes to innocuous stimuli. In clinical management of chronic pain, a bio-psycho-social model (Gatchel et al., 2007) is preferred since the above mentioned complex blend of factors - neurobiological, psychological (e.g., depression, catastrophizing, and anxiety), coping styles, and contextual factors - contribute to the development and maintenance of chronic pain (Alonso et al., 2004; Asmundson \& Katz, 2009; Börsbo et al., 2008; Dersh et al., 2001; Ericsson et al., 2002; MeansChristensen et al., 2008; Ocañez et al., 2010; Sofat et al., 2011; Sullivan et al., 2001).

\subsection{Central versus peripheral causes for chronic pain}

One of the consequences of the discovery of central sensitization is that CNS can change pain -e.g., amplification, duration, degree, and spatial extent - so that pain no longer directly reflects the peripheral noxious situation (Woolf, 2011). It is unknown whether a chronic pain condition can be driven by established central alterations such as central hyperexcitability, alterations in pain matrix, and alterations in descending mechanisms (facilitation) with very little or no peripheral stimuli or nociception. However, there are several indications that central alterations in nociceptive processing are driven by peripheral tissue alterations (Gerdle et al., 2008c) and peripheral nociceptive input (Schneider et al., 2010; Staud, 2010; Staud et al., 2009; Woolf, 2011).

\subsection{The microdialysis technique}

Concerns have been expressed at the lack of success in translating basic science data using animals into clinical analgesics (Lascelles \& Flecknell, 2010). Microdialysis may be able to 
replace animal experiments (Langley et al., 2008). The microdialysis technique offers a wellestablished in vivo method for studying the local biochemistry of individual tissues in the body (Ungerstedt, 1991), e.g., nociceptive and metabolic mechanisms. This technique has been used in neuroscience to monitor neurotransmitter release, but has also found application in monitoring the biochemistry of peripheral tissues in both animals and humans (Ungerstedt, 1991).

Microdialysis mimics the function of a capillary blood vessel by perfusing a thin dialysis tube (catheter) implanted into the tissue with a physiological saline solution. Through simple diffusion, substances can move across the dialysis membrane along the concentration gradient. The chemical analysis of the dialysate reveals the composition of the extracellular fluid. Thus microdialysis allows for continuous sampling of compounds in the interstitial space of the muscle, where nociceptor free nerve endings terminate close to the muscle fibres, providing accurate information on regional biochemical changes before such compounds are diluted and cleared by the circulatory system. The trapezius muscle has been used as a human model muscle for chronic myalgia both due to its clinical importance and to its accessibility for invasive investigations. Some studies use the masseter, vastus lateralis, and gastrocnemius muscles to examine myalgia.

To determine the concentrations of small molecules such as lactate, pyruvate, glutamate, and glucose, a catheter with a $20 \mathrm{kDa}$ cut-off is usually used (Waelgaard et al., 2006). To determine the concentrations of larger molecules such as cytokines, a catheter with a 100 kDa cut-off is usually used (Waelgaard et al., 2006).

A crucial parameter in microdialysis is relative recovery (RR): the ratio between substance concentrations in the dialysate to that in the perfusate (Afinowi et al., 2009; Dahlin et al., 2010 ; Ungerstedt, 1991). RR is used to determine the true concentration of extracellular fluid. Because the perfusate constantly flows across the membrane, a state of equilibrium will never be achieved and as a result the dialysate will only represent a certain percentage of the actual concentration of the extracellular fluid (Afinowi et al., 2009; Hamrin et al., 2002). Therefore, the final concentration in the dialysate partially depends on the flow rate of the perfusate. Low flow rate results in higher RR; high flow rates, result in lower RR. At a very low flow (i.e., $\leq 0.3 \mu \mathrm{l} / \mathrm{min}$ ), the recovery is near $100 \%$, but factors such as alterations in the osmotic pressure, temperature, weight cut-off, area of the membrane, concentration gradient, and composition of the perfusate can influence RR (Dahlin et al., 2010 ; Hamrin et al., 2002 ; Plock \& Kloft, 2005 ). Between cytokines have been reported marked variation in RR; molecular weight correlated negatively with RR (Helmy et al., 2009).

\subsection{Aim}

Microdialysis has several important advantages, but studies with patients are expensive and time consuming. Hence a systematic review of the literature is needed. Systematic knowledge of the results of such studies might help provide new assessment approaches of patients with chronic myalgia, new treatments, and new rehabilitation techniques for patients with chronic myalgia. Most research on muscle pain has been conducted on animals; however, this review will primarily focus on human studies of neck and shoulder myalgia, for which the frequently affected trapezius muscle often serves as a model muscle. This study systematically reviews studies in the literature that have investigated alterations 
in metabolic substances, pain-related substances (algesics), and anti-analgesics in different chronic muscle pain conditions (mainly myalgia) in humans using microdialysis. This systematic review was done to identify potential biomarkers - an objectively measured and evaluated indicator of, e.g., normal pathogenic processes (Ptolemy \& Rifai, 2010).

\section{Methods}

\subsection{Inclusion and exclusion criteria}

Studies that focused on chronic pain conditions affecting human muscles (myalgias) were included. To be included, the studies had to use microdialysis and had to use a patient group and a healthy control group, so articles concerning only healthy subjects have been excluded.

\subsection{Search strategy}

This review identified the studies fulfilling the above criteria in the systematic review of Larsson et al. (Larsson et al., 2007). Furthermore, we modified their search strategy in the following ways: ((muscle OR pain) AND microdialysis) OR (muscle AND pain AND induced) AND (Humans[Mesh] AND (Clinical Trial[ptyp] OR Meta-Analysis[ptyp] OR Review[ptyp]) AND English[lang] AND adult[MeSH] AND "last 10 years"[PDat]).

Using this strategy, PubMed was searched. From this search, the titles and abstracts were scrutinized. If the articles were relevant and necessary, they were read for further evaluation. We also checked reference lists of these articles. If the article was relevant according to our aim and inclusion criteria, we listed the results in tables. The tables of the different conditions reported the statistics concerning comparisons between the patient group and control group with respect to baseline data or corresponding data for all investigated substances. Moreover, we listed gender, flow rate, number of subjects in each group, and if and how the authors handled RR.

\subsection{Positive outcome with respect to potential biomarker}

A specific substance was classified as a potential biomarker if the majority of studies (including the majority of subjects) showed significantly lower or significantly higher concentrations in the patient group compared to the controls.

\section{Results}

Using the modified search strategy, we had 441 hits after searching PubMed. After screening, we identified 17 articles that fulfilled our inclusion criteria. Thirteen of these were not mentioned by Larsson et al. (Larsson et al., 2007). After scrutinizing these 13 articles and the articles selected by Larsson et al., we found 22 articles concerning different chronic pain conditions involving muscle. These articles are summarized in Tables 1-7. Moreover, we identified three articles concerning chronic tendinosis (Table 8).

\subsection{Chronic trapezius myalgia}

For chronic trapezius myalgia, we identified seven groups of patients reported in ten studies (Table 1). The majority of the studies reported increases in the interstitial concentrations of 
lactate (Flodgren et al., 2010; Flodgren et al., 2006; Larsson et al., 2008; Rosendal et al., 2004b; Sjogaard et al., 2010). However, the studies conducted by Flodgren et al. (Flodgren et al., 2010; Flodgren et al., 2006) did not compensate for relative recovery despite the fact that the flow rate was relatively high $(2 \mu \mathrm{l} / \mathrm{min})$ compared to a flow rate associated with full recovery $(0.3 \mu \mathrm{l} / \mathrm{min})$.

\begin{tabular}{|c|c|c|c|c|}
\hline Study & $\begin{array}{l}\text { Condition } \\
\text { Number of } \\
\text { Subjects, \% } \\
\text { women (W) } \\
\text { Muscles }\end{array}$ & $\begin{array}{l}\text { Substances } \\
\text { investigated } \\
\text { Flow rate }\end{array}$ & $\begin{array}{l}\text { Results } \\
\text { (Comparisons between } \\
\text { patients and HC; p-values) }\end{array}$ & \begin{tabular}{|l} 
Comments \\
(Compensated \\
for RR $(\mathrm{Y} / \mathrm{N})$ \\
or low flow \\
rate* \\
(NA or $\mathrm{Y} / \mathrm{N})$
\end{tabular} \\
\hline $\begin{array}{l}\text { Flodgren } \\
\text { et al. } \\
\text { (Flodgren } \\
\text { et al., 2005) }\end{array}$ & \begin{tabular}{|l|} 
Chronic shoulder \\
pain \\
$(\mathrm{CSP} ; \mathrm{n}=9)$, \\
$100 \% \mathrm{~W}$ \\
$\mathrm{HC}(\mathrm{n}=9)$, \\
$100 \% \mathrm{~W}$ \\
Trapezius
\end{tabular} & $\begin{array}{l}\text { Glutamate } \\
\text { PGE }_{2} \\
0.3 \mu \mathrm{l} / \mathrm{min}\end{array}$ & $\begin{array}{l}\text { Glutamate-trapezius: ns } \\
\text { PGE }_{2} \text {-trapezius: ns }\end{array}$ & $\begin{array}{l}\text { Compensated } \\
\text { for RR: } \mathrm{N} \\
\text { Low flow: } \mathrm{Y}\end{array}$ \\
\hline $\begin{array}{l}\text { Flodgren } \\
\text { et al. } \\
\text { (Flodgren } \\
\text { et al., 2010) }\end{array}$ & \begin{tabular}{|l|} 
Chronic trapezius \\
myalgia \\
(MYA; $\mathrm{n}=14)$, \\
$100 \% \mathrm{~W}$ \\
$\mathrm{HC}$ \\
(compared with \\
healthy subjects \\
$(\mathrm{n}=20)$ in \\
$($ Flodgren et al., \\
$2006))$, \\
$100 \% \mathrm{~W}$ \\
Trapezius
\end{tabular} & $\begin{array}{l}\text { Lactate } \\
\text { Pyruvate } \\
\text { Glutamate } \\
\text { PGE }_{2} \\
2 \mu \mathrm{l} / \mathrm{min}\end{array}$ & $\begin{array}{l}\text { Lactate-trapezius: ns } \\
\text { Pyruvate-trapezius: } \\
\text { Glutamate-trapezius: } \\
\text { MYA<HC, significant } \\
\text { PGE }_{2} \text {-trapezius: not } \\
\text { reported. }\end{array}$ & $\begin{array}{l}\text { Compensated } \\
\text { for RR: } \mathrm{N} \\
\text { Low flow: } \mathrm{N}\end{array}$ \\
\hline $\begin{array}{l}\text { Rosendal } \\
\text { et al. } \\
\text { (Rosendal } \\
\text { et al., } \\
\text { 2004b) }\end{array}$ & \begin{tabular}{|l|} 
Chronic trapezius \\
myalgia \\
$(\mathrm{MYA} ; \mathrm{n}=19)$, \\
$100 \% \mathrm{~W}$ \\
$\mathrm{HC}(\mathrm{n}=20)$, \\
$100 \% \mathrm{~W}$ \\
Trapezius
\end{tabular} & $\begin{array}{l}\text { Lactate } \\
\text { Pyruvate } \\
\text { Glutamate } \\
5 \text {-HT } \\
5 \mu \mathrm{l} / \mathrm{min}\end{array}$ & $\begin{array}{l}\text { Lactate-trapezius: } \\
\text { MYA > HC; } P=0.001 \\
\text { Pyruvate-trapezius: } \\
\text { MYA > HC; } P=0.001 \\
\text { Glutamate-trapezius: } \\
\text { MYA > HC; } P=0.05 \\
\text { 5-HT-trapezius: } \\
\text { MYA > HC; } P=0.01 \\
\end{array}$ & $\begin{array}{l}\text { Compensated } \\
\text { for RR: } Y \\
\text { Low flow: NA }\end{array}$ \\
\hline $\begin{array}{l}\text { Rosendal } \\
\text { et al. } \\
\text { (Rosendal } \\
\text { et al., 2005) }\end{array}$ & \begin{tabular}{|l|} 
Chronic trapezius \\
myalgia \\
$(\mathrm{MYA} ; \mathrm{n}=19)$, \\
$100 \% \mathrm{~W}$ \\
$\mathrm{HC}(\mathrm{n}=20)$, \\
$100 \% \mathrm{~W}$ \\
Trapezius
\end{tabular} & $\begin{array}{l}\mathrm{K}^{+} \\
\mathrm{LDH} \\
\mathrm{IL}-6 \\
\text { Collagen } \\
\text { turnover } \\
5 \mu \mathrm{l} / \mathrm{min}\end{array}$ & $\begin{array}{l}\text { K+-trapezius: } \\
\text { MYA > HC; } \\
\text { P=significant } \\
\text { LDH-trapezius: ns } \\
\text { IL-6-trapezius: ns } \\
\text { Collagen turnover- } \\
\text { trapezius: ns }\end{array}$ & $\begin{array}{l}\text { Compensated } \\
\text { for RR: Y } \\
\text { Low flow: NA }\end{array}$ \\
\hline
\end{tabular}




\begin{tabular}{|c|c|c|c|c|}
\hline Study & \begin{tabular}{|l} 
Condition \\
Number of \\
Subjects, \% \\
women (W) \\
Muscles
\end{tabular} & $\begin{array}{l}\text { Substances } \\
\text { investigated } \\
\text { Flow rate }\end{array}$ & $\begin{array}{l}\text { Results } \\
\text { (Comparisons between } \\
\text { patients and HC; p-values) }\end{array}$ & \begin{tabular}{|l} 
Comments \\
(Compensated \\
for RR $(\mathrm{Y} / \mathrm{N})$ \\
or low flow \\
rate* \\
(NA or $\mathrm{Y} / \mathrm{N})$
\end{tabular} \\
\hline $\begin{array}{l}\text { Gerdle } \\
\text { et al. } \\
\text { (Gerdle et } \\
\text { al., 2008b) }\end{array}$ & $\begin{array}{l}\text { Chronic trapezius } \\
\text { myalgia } \\
(\text { MYA; } n=19), \\
100 \% W \\
\text { Chronic whiplash } \\
\text { associated } \\
\text { disorders } \\
(\text { WAD; } n=22), \\
100 \% W \\
\text { HC }(n=20), \\
100 \% W \\
\text { Trapezius }\end{array}$ & $\begin{array}{l}\text { BKN } \\
\text { Kallidin } \\
5 \mu \mathrm{l} / \mathrm{min}\end{array}$ & $\begin{array}{l}\text { BKN-trapezius: } \\
\text { No group differences. } \\
\text { Kallidin-trapezius: } \\
\text { MYA>HC; } \\
\text { P=0.018 } \\
\text { No other group differences } \\
\text { for this substance. }\end{array}$ & $\begin{array}{l}\text { Compensated } \\
\text { for RR: } Y \\
\text { Low flow: NA }\end{array}$ \\
\hline \begin{tabular}{|l|} 
Larsson \\
et al. \\
(Larsson et \\
al., 2008)
\end{tabular} & $\begin{array}{l}\text { Chronic trapezius } \\
\text { myalgia } \\
(\mathrm{MYA} ; \mathrm{n}=20), \\
100 \% \mathrm{~W} \\
\mathrm{HC}(\mathrm{n}=20), \\
100 \% \mathrm{~W} \\
\text { Trapezius }\end{array}$ & $\begin{array}{l}\text { Lactate } \\
\text { Pyruvate } \\
\text { Glutamate } \\
5-H T \\
\mathrm{~K}^{+} \\
\text {BKN } \\
\text { GM-CSF } \\
\text { IL-1 } \beta \\
\text { IL-6 } \\
\text { IL-8 } \\
\text { TNF-a } \\
\text { IL-2 } \\
\text { IL-4 } \\
\text { IL-5 } \\
\text { IL-10 } \\
5 \mu l / m i n\end{array}$ & $\begin{array}{l}\text { Lactate-trapezius: ns } \\
\text { Pyruvate-trapezius: } \\
\text { MYA > HC; P=0.032 } \\
\text { Glutamate-trapezius: } \\
\text { MYA > HC; P=0.005 } \\
\text { 5-HT-trapezius: } \\
\text { MYA > HC; P=0.023 } \\
\text { K+-trapezius: ns } \\
\text { BKN- trapezius: ns } \\
\text { GM-CSF- trapezius: ns } \\
\text { IL-1 } \beta \text {-trapezius: ns } \\
\text { IL-6-trapezius: ns } \\
\text { IL-8-trapezius: ns } \\
\text { TNF-a-trapezius: ns } \\
\text { IL-2-trapezius: ns } \\
\text { IL-4-trapezius: ns } \\
\text { IL-5-trapezius: ns } \\
\text { IL-10-trapezius: ns }\end{array}$ & $\begin{array}{l}\text { Compensated } \\
\text { for RR: Y } \\
\text { Low flow: NA }\end{array}$ \\
\hline $\begin{array}{l}\text { Ghafouri } \\
\text { et al. } \\
\text { (Ghafouri } \\
\text { et al., 2010) }\end{array}$ & $\begin{array}{l}\text { Chronic } \\
\text { trapezius } \\
\text { myalgia, } \\
(\mathrm{MYA} \text {; }=18), \\
100 \% \mathrm{~W} \\
\mathrm{HC}(\mathrm{n}=30), \\
100 \% \mathrm{~W} \\
\\
\text { Trapezius }\end{array}$ & $\begin{array}{l}5-\mathrm{HT} \\
5 \mu \mathrm{l} / \mathrm{min}\end{array}$ & $\begin{array}{l}\text { 5-HT- trapezius: } \mathrm{MYA}>\mathrm{HC} \\
\mathrm{P}=0.044\end{array}$ & $\begin{array}{l}\text { Compensated } \\
\text { for RR: Y } \\
\text { Low flow: NA }\end{array}$ \\
\hline
\end{tabular}




\begin{tabular}{|c|c|c|c|c|}
\hline Study & \begin{tabular}{|l} 
Condition \\
Number of \\
Subjects, \% \\
women (W) \\
Muscles
\end{tabular} & $\begin{array}{l}\text { Substances } \\
\text { investigated } \\
\text { Flow rate }\end{array}$ & $\begin{array}{l}\text { Results } \\
\text { (Comparisons between } \\
\text { patients and HC; p-values) }\end{array}$ & \begin{tabular}{|l} 
Comments \\
(Compensated \\
for RR $(\mathrm{Y} / \mathrm{N})$ \\
or low flow \\
rate* \\
(NA or $\mathrm{Y} / \mathrm{N})$
\end{tabular} \\
\hline $\begin{array}{l}\text { Ghafouri } \\
\text { et al. } \\
\text { (Ghafouri } \\
\text { et al., 2011) }\end{array}$ & $\begin{array}{l}\text { Chronic trapezius } \\
\text { myalgia, } \\
(\mathrm{MYA} ; \mathrm{n}=11), \\
100 \% \mathrm{~W} \\
\mathrm{HC}(\mathrm{n}=11), \\
100 \% \mathrm{~W} \\
\text { Trapezius }\end{array}$ & $\begin{array}{l}\text { PEA } \\
\text { SEA } \\
5 \mu 1 / \min \end{array}$ & $\begin{array}{l}\text { PEA-trapezius: } \\
\text { MYA>HC, } P=0.011 \\
\text { SEA-trapezius: } \\
\text { MYA }>\mathrm{HC}, \mathrm{P}=0.002 \\
\text { (The statistics are for } \\
\text { dialysate concentrations, but } \\
\text { significant differences were } \\
\text { found when compensated } \\
\text { for } \mathrm{RR} \text { ) }\end{array}$ & $\begin{array}{l}\text { Compensated } \\
\text { for RR: Y } \\
\text { Low flow: NA }\end{array}$ \\
\hline \begin{tabular}{|l|} 
Sjøgaard \\
et al \\
(Sjogaard \\
et al., 2010)
\end{tabular} & \begin{tabular}{|l|} 
Chronic trapezius \\
myalgia (MYA; \\
n=43), 100\%W \\
HC $(n=19)$ \\
$100 \% W$ \\
Trapezius
\end{tabular} & $\begin{array}{l}\text { Lactate } \\
\text { Pyruvate } \\
\text { Glucose } \\
\mathrm{K}^{+} \\
5 \mu \mathrm{l} / \mathrm{min}\end{array}$ & $\begin{array}{l}\text { Lactate-trapezius: } \\
\text { MYA>HC, significant } \\
\text { Pyruvate-trapezius: } \\
\text { MYA>HC, significant } \\
\text { Glucose-trapezius: ns } \\
\mathrm{K}^{+} \text {-trapezius: ns }\end{array}$ & $\begin{array}{l}\text { Compensated } \\
\text { for RR: Y } \\
\text { Low flow: NA }\end{array}$ \\
\hline $\begin{array}{l}\text { Shah } \\
\text { et al } \\
\text { (Shah et } \\
\text { al., 2005) }\end{array}$ & $\begin{array}{l}\text { Myofascial } \\
\text { trapezius pain } \\
\text { with active } \\
\text { trigger point } \\
\text { (MFactive; } \mathrm{n}=3 \text { ) } \\
\text { HC with latent } \\
\text { trigger point } \\
\text { (HClatent; } \mathrm{n}=3 \text { ) } \\
\text { HC without } \\
\text { trigger point } \\
(\mathrm{HC} ; \mathrm{n}=3) \\
\text { Trigger points of } \\
\text { Trapezius }\end{array}$ & $\begin{array}{l}\text { BKN } \\
\text { CGRP } \\
\text { Substance P } \\
\text { IL-1 } \beta \\
\text { TNF-a } \\
\text { 5-HT } \\
\text { Norepineph } \\
\text { rine } \\
\mathrm{H}^{+} \\
\text {1and } 2 \\
\mu \mathrm{l} / \mathrm{min}\end{array}$ & 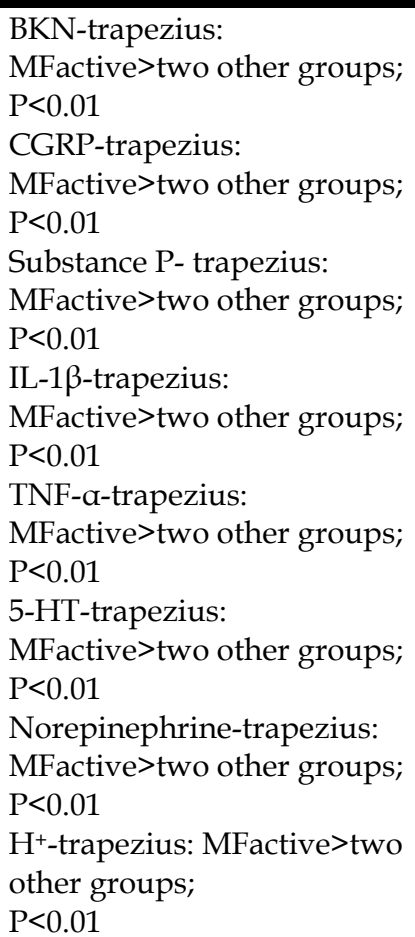 & $\begin{array}{l}\text { Compensated } \\
\text { for RR: Y } \\
\text { Low flow: NA }\end{array}$ \\
\hline
\end{tabular}




\begin{tabular}{|c|c|c|c|c|}
\hline Study & \begin{tabular}{|l} 
Condition \\
Number of \\
Subjects, \% \\
women (W) \\
Muscles
\end{tabular} & $\begin{array}{l}\text { Substances } \\
\text { investigated } \\
\text { Flow rate }\end{array}$ & $\begin{array}{l}\text { Results } \\
\text { (Comparisons between } \\
\text { patients and HC; p-values) }\end{array}$ & \begin{tabular}{|l} 
Comments \\
(Compensated \\
for RR $(\mathrm{Y} / \mathrm{N})$ \\
or low flow \\
rate* \\
(NA or $\mathrm{Y} / \mathrm{N})$
\end{tabular} \\
\hline $\begin{array}{l}\text { Shah et al. } \\
\text { (Shah et } \\
\text { al., 2008) }\end{array}$ & $\begin{array}{l}\text { Myofascial } \\
\text { trapezius pain } \\
\text { with active } \\
\text { trigger point } \\
\text { (MFactive; } \mathrm{n}=3 \text { ) } \\
\text { HC with latent } \\
\text { trigger point } \\
\text { (HClatent; } \mathrm{n}=3 \text { ) } \\
\text { HC without } \\
\text { trigger point } \\
\text { (HC; } \mathrm{n}=3 \text { ) } \\
\text { Trigger points of } \\
\text { Trapezius } \\
\text { Gastrocnemius } \\
\text { medialis without } \\
\text { trigger points }\end{array}$ & $\begin{array}{l}\text { BKN } \\
\text { CGRP } \\
\text { Substance P } \\
\text { IL-1 } \beta \\
\text { TNF-a } \\
\text { IL-6 } \\
\text { IL-8 } \\
5-H T \\
\text { Norepineph } \\
\text { rine } \\
\mathrm{H}^{+} \\
1 \text { and } \\
2 \mu \mathrm{l} / \mathrm{min}\end{array}$ & 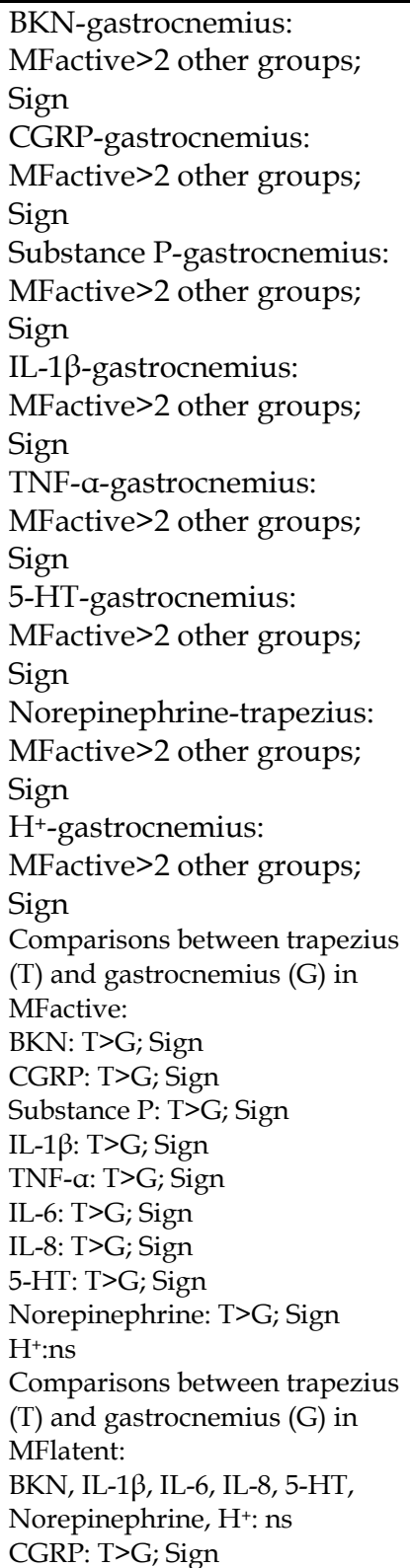 & $\begin{array}{l}\text { Compensated } \\
\text { for RR: } Y \\
\text { Low flow: NA }\end{array}$ \\
\hline
\end{tabular}




\begin{tabular}{|c|c|c|c|c|}
\hline Study & $\begin{array}{l}\text { Condition } \\
\text { Number of } \\
\text { Subjects, \% } \\
\text { women }(W) \\
\text { Muscles }\end{array}$ & $\begin{array}{l}\text { Substances } \\
\text { investigated } \\
\text { Flow rate }\end{array}$ & $\begin{array}{l}\text { Results } \\
\text { (Comparisons between } \\
\text { patients and HC; p-values) }\end{array}$ & \begin{tabular}{|l|} 
Comments \\
(Compensated \\
for RR $(\mathrm{Y} / \mathrm{N})$ \\
or low flow \\
rate* \\
$(\mathrm{NA}$ or $\mathrm{Y} / \mathrm{N})$
\end{tabular} \\
\hline & & & $\begin{array}{l}\text { Substance P: T>G; Sign } \\
\text { TNF-a: T>G; Sign } \\
\text { Comparisons between trapezius } \\
(\mathrm{T}) \text { and gastrocnemius }(G) \text { in } \\
\text { HC: } \\
\text { BKN: T>G; Sign } \\
\text { Other substances: ns }\end{array}$ & \\
\hline
\end{tabular}

*Low flow rate associated with $100 \%$ relative recovery was defined as $0.3 \mu 1 / \mathrm{min}$.

Table 1. Studies of chronic trapezius myalgia. The results of the different substances are baseline data. Healthy controls are abbreviated as HC. The bold horizontal lines indicate different patient groups.

A similar pattern was noted for the majority of studies reporting increased interstitial concentrations of pyruvate (Flodgren et al., 2010; Flodgren et al., 2006; Larsson et al., 2008; Rosendal et al., 2004b; Sjogaard et al., 2010). Flodgren et al. did not show any significant differences for pyruvate (Flodgren et al., 2010; Flodgren et al., 2006).

Three studies that focused on chronic trapezius myalgia investigated the interstitial muscle concentration of glutamate (Flodgren et al., 2005; Larsson et al., 2008; Rosendal et al., 2004b). In two of these studies (Larsson et al., 2008; Rosendal et al., 2004b), which were markedly larger than the third study (Flodgren et al., 2005), revealed significant increases in the interstitial concentrations of glutamate. In all the studies investigating glutamate, the interstitial muscle concentration of 5-HT was significantly increased (Ghafouri et al., 2010; Larsson et al., 2008; Rosendal et al., 2004b; Shah et al., 2008; Shah et al., 2005). Furthermore, glutamate was also increased in patients with chronic WAD (Table 2) (Gerdle et al., 2008c).

Four studies examined the interstitial concentrations of BKN and/or Kallidin (Gerdle et al., 2008b; Larsson et al., 2008; Shah et al., 2008; Shah et al., 2005). Shah et al. (Shah et al., 2008; Shah et al., 2005) found increased levels of BKN in subjects with active trigger points and the levels were higher in the trapezius (with pain) than in a pain-free distant muscle. In contrast, two studies - a field study and a laboratory study - found no differences between patients and controls (Gerdle et al., 2008b; Larsson et al., 2008). Kallidin was only investigated in one study and this study reported significantly higher interstitial levels in patients with chronic trapezius myalgia (Gerdle et al., 2008b).

Several studies have investigated cytokines, but only Shah et al. found significant differences for active trigger points (Shah et al., 2008; Shah et al., 2005). These authors also have compared the levels of cytokines in the myalgic trapezius (trigger points) with a muscle without pain and found higher levels in the aching muscle. These studies, however, are limited because their sample size was small. Larger studies have not found elevated levels of cytokines (Larsson et al., 2008; Rosendal et al., 2005). In three relatively large 
studies, potassium revealed no consistent pattern (Larsson et al., 2008; Rosendal et al., 2005; Sjogaard et al., 2010). PGE 2 was not increased in two groups of patients (Flodgren et al., 2005; Flodgren et al., 2010; Flodgren et al., 2006), but the first of these groups was relatively small (Flodgren et al., 2005) and the other had possible methodological drawbacks related to RR (Flodgren et al., 2010; Flodgren et al., 2006).

\begin{tabular}{|c|c|c|c|c|}
\hline Study & $\begin{array}{l}\text { Condition } \\
\text { Number of } \\
\text { Subjects, \% } \\
\text { women (W) } \\
\text { Muscles }\end{array}$ & $\begin{array}{l}\text { Substances } \\
\text { investigated } \\
\text { Flow rate }\end{array}$ & $\begin{array}{l}\text { Results } \\
\text { (Comparisons between } \\
\text { patients and } \mathrm{HC} ; \\
\text { p-values) }\end{array}$ & $\begin{array}{l}\text { Comments } \\
\text { (Compensated for } \\
\text { RR }(\mathrm{Y} / \mathrm{N}) \text { or low } \\
\text { flow rate* } \\
(\mathrm{NA} \text { or } \mathrm{Y} / \mathrm{N})\end{array}$ \\
\hline $\begin{array}{l}\text { Gerdle } \\
\text { et al. } \\
\text { (Gerdle et } \\
\text { al., 2008c) }\end{array}$ & $\begin{array}{l}\text { Chronic } \\
\text { whiplash } \\
\text { associated } \\
\text { disorders } \\
(\text { WAD; } n=22) \text {, } \\
100 \% \text { W } \\
\text { HC }(n=20), \\
100 \% \text { W } \\
\text { Trapezius }\end{array}$ & \begin{tabular}{|l} 
Lactate \\
Pyruvate \\
Glutamate \\
$\mathrm{K}^{+}$ \\
$5-\mathrm{HT}$ \\
$\mathrm{IL}-6$ \\
\\
$5 \mu \mathrm{l} / \mathrm{min}$
\end{tabular} & $\begin{array}{l}\text { Lactate-trapezius: } \mathrm{ns} \\
\text { Pyruvate-trapezius: ns } \\
\text { Glutamate-trapezius: ns } \\
\text { K+- trapezius: ns } \\
\text { 5-HT-trapezius: } \\
\text { WAD>HC; } \mathrm{P}=0.05 \\
\text { IL-6-trapezius: } \\
\text { WAD>HC; } \mathrm{P}=0.008\end{array}$ & $\begin{array}{l}\text { Compensated for } \\
\text { RR: Y } \\
\text { Low flow: NA }\end{array}$ \\
\hline $\begin{array}{l}\text { Gerdle } \\
\text { et al. } \\
\text { (Gerdle et } \\
\text { al., 2008b) }\end{array}$ & 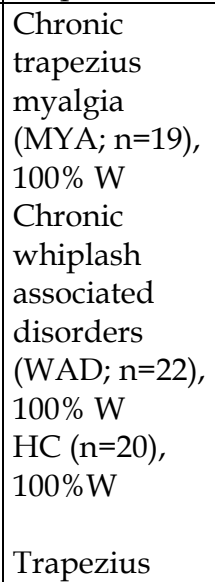 & $\begin{array}{l}\text { BKN } \\
\text { Kallidin } \\
5 \mu \mathrm{l} / \mathrm{min}\end{array}$ & $\begin{array}{l}\text { BKN- trapezius: No } \\
\text { group differences. } \\
\text { Kallidin-trapezius: } \\
\text { MYA>HC; } P=0.018 \\
\text { No other group } \\
\text { differences for this } \\
\text { substance. }\end{array}$ & $\begin{array}{l}\text { Compensated for } \\
\text { RR: Y } \\
\text { Low flow: NA }\end{array}$ \\
\hline
\end{tabular}

*Low flow rate associated with $100 \%$ relative recovery was defined as $0.3 \mu \mathrm{l} / \mathrm{min}$.

Table 2. Studies of chronic WAD. The results of the different substances are baseline data. Healthy controls are abbreviated as HC.

In two studies, Shah et al. investigated P and CGRP (Shah et al., 2008; Shah et al., 2005). They found significant increases in active trigger points in the trapezius and found that these levels were higher in the aching trapezius than in a distant pain-free muscle.

Substances only reported in single studies/patient groups were glucose (no difference) (Sjogaard et al., 2010), norepinephrine (significant difference) (Shah et al., 2008; Shah et al., 2005), and $\mathrm{H}^{+}$(significant difference) (Shah et al., 2008; Shah et al., 2005). 


\subsection{Chronic WAD}

We identified two studies (Table 2) using the same material concerning patients with chronic WAD (Gerdle et al., 2008b; Gerdle et al., 2008c), and 5-HT and IL-6 were significantly higher in WAD than in HC. No differences were found in the concentrations of investigated metabolites, potassium, glutamate, $\mathrm{BKN}$, or kallidin compared with healthy controls.

\subsection{Fibromyalgia}

Two studies investigated fibromyalgia patients (Table 3). In the larger study investigating the painful trapezius muscle of fibromyalgia patients, significantly higher interstitial concentrations of lactate and pyruvate were found compared to healthy controls (Gerdle et al., 2010). In the other study, the vastus lateralis muscle was investigated in eight patients with fibromyalgia and the authors reported no differences in concentrations of lactate compared to controls (McIver et al., 2006). It is unclear from this study if the vastus lateralis was habitually painful and/or painful at palpation (e.g., tender point examination). Moreover, no compensation for RR was done despite a relatively high flow rate.

\begin{tabular}{|c|c|c|c|c|}
\hline Study & $\begin{array}{l}\text { Condition } \\
\text { Number of } \\
\text { Subjects, \% } \\
\text { women (W) } \\
\text { Muscles } \\
\end{array}$ & $\begin{array}{l}\text { Substances } \\
\text { investigated } \\
\text { Flow rate }\end{array}$ & $\begin{array}{l}\text { Results } \\
\text { (Comparisons between } \\
\text { patients and HC; } \\
\text { p-values) }\end{array}$ & $\begin{array}{l}\text { Comments } \\
\text { (Compensated } \\
\text { for RR }(\mathrm{Y} / \mathrm{N}) \text { or } \\
\text { low flow rate* } \\
(\mathrm{NA} \text { or } \mathrm{Y} / \mathrm{N})\end{array}$ \\
\hline $\begin{array}{l}\text { Gerdle } \\
\text { et al. } \\
\text { (Gerdle et } \\
\text { al., 2010) }\end{array}$ & $\begin{array}{l}\text { Fibromyalgia } \\
(\mathrm{FM} ; \mathrm{n}=19), \\
100 \% \mathrm{~W} \\
\mathrm{HC}(\mathrm{n}=19), \\
100 \% \mathrm{~W} \\
\text { Trapezius }\end{array}$ & $\begin{array}{l}\text { Lactate } \\
\text { Pyruvate } \\
\text { Glutamate } \\
\\
0.3 \mu \mathrm{l} / \mathrm{min}\end{array}$ & $\begin{array}{l}\text { Lactate-trapezius: } \\
\text { FM>HC; } \mathrm{P}=0.039 \\
\text { Pyruvate-trapezius: } \\
\text { FM>HC; } \mathrm{P}=0.001 \\
\text { Glutamate-trapezius: } \\
\text { ns. }\end{array}$ & $\begin{array}{l}\text { Compensated for } \\
\text { RR: } \mathrm{N} \\
\text { Low flow: } \mathrm{Y}\end{array}$ \\
\hline $\begin{array}{l}\text { McIver } \\
\text { et al } \\
\text { (McIver et } \\
\text { al., 2006) }\end{array}$ & $\begin{array}{l}\text { Fibromyalgia } \\
(\mathrm{FM} ; \mathrm{n}=8), \\
100 \% \mathrm{~W} \\
\mathrm{HC}(\mathrm{n}=8), \\
100 \% \mathrm{~W} \\
\text { Vastus lateralis }\end{array}$ & $\begin{array}{l}\text { Lactate } \\
2 \mu \mathrm{l} / \mathrm{min}\end{array}$ & Lactate-vastus: ns & $\begin{array}{l}\text { Compensated for } \\
\text { RR: } \mathrm{N} \\
\text { Low flow: } \mathrm{N}\end{array}$ \\
\hline
\end{tabular}

*Low flow rate associated with $100 \%$ relative recovery was defined as $0.3 \mu 1 / \mathrm{min}$

Table 3. Studies of fibromyalgia. The results of the different substances are baseline data. Healthy controls are abbreviated as HC.

\subsection{Temporomandibular pain disorders}

Compared to the healthy controls, fibromyalgia patients had increased 5-HT in the masseter muscle (Ernberg et al., 1999). Compared to the healthy controls, myofascial temporomandibular disorder patients had significantly higher glutamate levels (Castrillon et al., 2010) (Table 4). 


\begin{tabular}{|c|c|c|c|c|}
\hline Study & $\begin{array}{l}\text { Condition } \\
\text { Number of Subjects, } \\
\% \text { women (W) } \\
\text { Muscles }\end{array}$ & $\begin{array}{l}\text { Substances } \\
\text { investigated } \\
\text { Flow rate }\end{array}$ & $\begin{array}{l}\text { Results } \\
\text { (Comparisons } \\
\text { between patients } \\
\text { and HC; p-values) }\end{array}$ & $\begin{array}{l}\text { Comments } \\
\text { (Compensated } \\
\text { for RR }(\mathrm{Y} / \mathrm{N}) \text { or } \\
\text { low flow rate* } \\
(\mathrm{NA} \text { or } \mathrm{Y} / \mathrm{N}) \\
\end{array}$ \\
\hline \begin{tabular}{|l|} 
Ernberg \\
et al \\
(Ernberg et \\
al., 1999)
\end{tabular} & $\begin{array}{l}\text { Fibromyalgia } \\
(\mathrm{FM} ; \mathrm{n}=18), \\
100 \% \mathrm{~W} \\
\text { Localized myalgia of } \\
\text { the } \\
\text { temporomandibular } \\
\text { system } \\
(\mathrm{LM} ; \mathrm{n}=17), 76 \% \mathrm{~W} \\
\text { HC }(\mathrm{n}=10), 60 \% \mathrm{~W} \\
\text { Masseter }\end{array}$ & $\begin{array}{l}\text { 5-HT } \\
\text { (corrected for } \\
\text { S-5-HT) } \\
7 \mu \mathrm{l} / \mathrm{min}\end{array}$ & $\begin{array}{l}\text { 5-HT } \\
\text { (corrected for S-5- } \\
\text { HT)-trauma- } \\
\text { masseter: } \\
\text { FM>HC: } \\
\mathrm{P}=0.05 \\
5-\mathrm{HT} \\
\text { (corrected for S-5- } \\
\text { HT)-baseline- } \\
\text { masseter: no } \\
\text { group differences }\end{array}$ & $\begin{array}{l}\text { Compensated for } \\
\text { RR: N, but } \\
\text { corrected for S- } \\
\text { 5-HT } \\
\text { Low flow:N }\end{array}$ \\
\hline $\begin{array}{l}\text { Hedenberg- } \\
\text { Magnusson } \\
\text { et al } \\
\text { (Hedenberg- } \\
\text { Magnusson } \\
\text { et al., 2001) }\end{array}$ & $\begin{array}{l}\text { Fibromyalgia } \\
(\mathrm{FM} ; \mathrm{n}=19), \\
89 \% \mathrm{~W} \\
\text { Localized myalgia of } \\
\text { the } \\
\text { temporomandibular } \\
\text { system } \\
(\mathrm{LM} ; \mathrm{n}=19), 74 \% \mathrm{~W} \\
\text { HC }(\mathrm{n}=11), 64 \% \mathrm{~W} \\
\text { Masseter }\end{array}$ & $\begin{array}{l}\mathrm{PGE}_{2} \\
\text { Leukotriene } \\
\mathrm{B}_{4}(\mathrm{LTB} 4) \\
7 \mu \mathrm{l} / \mathrm{min}\end{array}$ & $\begin{array}{l}\text { PGE }{ }_{2} \text {-masseter: ns } \\
\text { LTB4-masseter: } \\
\text { FM>LM; } P=0.05\end{array}$ & $\begin{array}{l}\text { Compensated for } \\
\text { RR: } \mathrm{N} \\
\text { Low flow: } \mathrm{N}\end{array}$ \\
\hline $\begin{array}{l}\text { Castrillon } \\
\text { (Castrillon et } \\
\text { al., 2010) }\end{array}$ & $\begin{array}{l}\text { Myofascial } \\
\text { temporomandibular } \\
\text { disorder pain } \\
(\mathrm{TMD} ; \mathrm{n}=13), 77 \% \mathrm{~W} \\
\mathrm{HC}(\mathrm{n}=10), 80 \% \mathrm{~W} \\
\text { Masseter }\end{array}$ & $\begin{array}{l}\text { Glutamate } \\
2 \mu \mathrm{l} / \mathrm{min}\end{array}$ & $\begin{array}{l}\text { Glutamate- } \\
\text { masseter: } \\
\text { TMD> HC; } \\
\mathrm{P}=0.023\end{array}$ & $\begin{array}{l}\text { Compensated for } \\
\text { RR: } \\
\text { Low flow: }\end{array}$ \\
\hline
\end{tabular}

*Low flow rate associated with $100 \%$ relative recovery was defined as $0.3 \mu \mathrm{l} / \mathrm{min}$.

Table 4. Studies of temporomandibular pain disorders. The results of the different substances are baseline data. Healthy controls are abbreviated as HC.

\subsection{Chronic tension type headaches}

In two studies of one group of patients (Ashina et al., 2002; Ashina et al., 2003) with chronic tension headaches, no differences were found in metabolites and some algesic substances (e.g., BKN and PGE2) in the trapezius muscle (Table 5).

\subsection{Polymyalgia rheumatica}

We found two studies of polymyalgia rheumatica based on the same groups of subjects (Kreiner \& Galbo, 2011; Kreiner et al., 2010) (Table 6). These two studies reported marked 
alterations in cytokines but also to some extent in algesic substances such as glutamate in two muscles. These two studies also reported 5-HT in one muscle and in BKN in one muscle. Interestingly, all alterations were normalized after prednisolone treatment.

\begin{tabular}{|c|c|c|c|c|}
\hline Study & $\begin{array}{l}\text { Condition } \\
\text { Number of Subjects, } \\
\% \text { women }(\mathrm{W}) \\
\text { Muscles }\end{array}$ & $\begin{array}{l}\text { Substances } \\
\text { investigated } \\
\text { Flow rate }\end{array}$ & $\begin{array}{l}\text { Results } \\
\text { (Comparisons } \\
\text { between patients } \\
\text { and HC; } \\
\text { p-values) }\end{array}$ & $\begin{array}{l}\text { Comments } \\
\text { (Compensated } \\
\text { for RR }(\mathrm{Y} / \mathrm{N}) \\
\text { or low flow } \\
\text { rate* } \\
(\mathrm{NA} \text { or } \mathrm{Y} / \mathrm{N})\end{array}$ \\
\hline $\begin{array}{l}\text { Ashina } \\
\text { et al. } \\
\text { (Ashina et } \\
\text { al., 2003) }\end{array}$ & $\begin{array}{l}\text { Chronic tension-type } \\
\text { Headache } \\
\text { (CTTH; } n=16), \\
63 \% \mathrm{~W} \\
\mathrm{HC} \\
(\mathrm{n}=17), \\
71 \% \mathrm{~W} \\
\text { Trapezius }\end{array}$ & $\begin{array}{l}\text { Pyruvate } \\
\text { Glucose } \\
\text { Urea } \\
\text { Glutamate } \\
\mathrm{K}^{+} \\
\mathrm{BKN} \\
\mathrm{PGE}_{2} \\
\mathrm{ATP} \\
2 \mu \mathrm{l} / \mathrm{min}\end{array}$ & $\begin{array}{l}\text { Pyruvate-trapezius: } \\
\text { ns } \\
\text { Glucose-trapezius: } \\
\text { ns } \\
\text { Urea-trapezius: ns } \\
\text { Glutamate- } \\
\text { trapezius: ns } \\
\mathrm{K}^{+} \text {-trapezius: ns } \\
\text { BKN-trapezius: ns } \\
\text { PGE -trapezius: ns } \\
\text { ATP-trapezius: } n s\end{array}$ & $\begin{array}{l}\text { Compensated for } \\
\text { RR: Y } \\
\text { Low flow: NA }\end{array}$ \\
\hline $\begin{array}{l}\text { Ashina } \\
\text { et al. } \\
\text { (Ashina et } \\
\text { al., 2002) }\end{array}$ & $\begin{array}{l}\text { Chronic tension-type } \\
\text { Headache } \\
(\mathrm{CTTH} ; \\
\mathrm{n}=16), \\
63 \% \mathrm{~W} \\
\mathrm{HC}(\mathrm{n}=17), \\
71 \% \mathrm{~W} \\
\text { Trapezius }\end{array}$ & $\begin{array}{l}\text { Lactate } \\
2 \mu \mathrm{l} / \mathrm{min}\end{array}$ & $\begin{array}{l}\text { Lactate- trapezius: } \\
\text { ns }\end{array}$ & $\begin{array}{l}\text { Compensated for } \\
\text { RR: Y } \\
\text { Low flow: NA }\end{array}$ \\
\hline
\end{tabular}

* Low flow rate associated with $100 \%$ relative recovery was defined as $0.3 \mu \mathrm{l} / \mathrm{min}$.

Table 5. Studies of chronic tension type headaches. The results of the different substances are baseline data. Healthy controls are abbreviated as HC.

\subsection{Mitochondrial myopathy}

In a study of mitochondrial myopathy that included four patients, no significant differences were found for metabolites, glutamate, hypoxanthine, urate, and aspartate (Table 7).

\subsection{Chronic tendinosis}

We also found three studies that focused on chronic tendinosis (Table 8). These three studies are small and have investigated different tendons with pain. The interstitial concentrations of glutamate, $\mathrm{PGE}_{2}$, and lactate were investigated, but no consistent pattern with respect to these substances was found. 


\begin{tabular}{|c|c|c|c|c|}
\hline Study & $\begin{array}{l}\text { Condition } \\
\text { Number of } \\
\text { Subjects, \% } \\
\text { women(W) } \\
\text { Muscles }\end{array}$ & $\begin{array}{l}\text { Substances } \\
\text { investigated } \\
\text { Flow rate }\end{array}$ & $\begin{array}{l}\text { Results } \\
\text { (comparisons between patients } \\
\text { and } \mathrm{HC} ; \mathrm{p} \text {-values) }\end{array}$ & $\begin{array}{l}\text { Comments } \\
\text { (Compensated } \\
\text { for RR }(\mathrm{Y} / \mathrm{N}) \text { or } \\
\text { low flow rate* } \\
(\mathrm{NA} \text { or } \mathrm{Y} / \mathrm{N})\end{array}$ \\
\hline $\begin{array}{l}\text { Kreiner \& } \\
\text { Galbo } \\
\text { (Kreiner \& } \\
\text { Galbo, } \\
\text { 2011) }\end{array}$ & 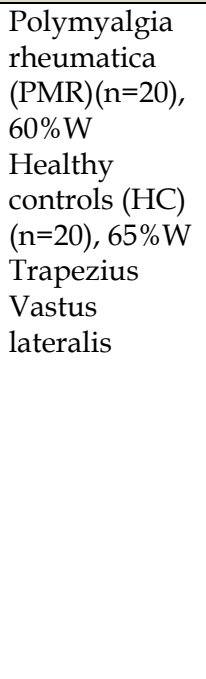 & $\begin{array}{l}\text { Lactate } \\
\text { Pyruvate } \\
\text { Glutamate } \\
5 \text {-HT } \\
\text { BKN } \\
\text { PGE2 } \\
\text { K+ } \\
\text { ATP } \\
3 \mu \mathrm{l} / \text { min }\end{array}$ & $\begin{array}{l}\text { Lactate -trapezius: PMR >HC; } \\
\text { P=0.05 } \\
\text { Pyruvate -trapezius: ns } \\
\text { Glutamate-trapezius: PMR } \\
\text { >HC; P=0.05 } \\
\text { 5-HT-trapezius: ns } \\
\text { BKN-trapezius: ns } \\
\text { PGE2-trapezius: ns } \\
\text { K+-trapezius: ns } \\
\text { ATP-trapezius: ns } \\
\text { Lactate-vastus: ns } \\
\text { Pyruvate-vastus: ns } \\
\text { Glutamate-vastus: PMR >HC; } \\
\text { P=0.05 } \\
\text { 5-HT-vastus: PMR >HC; } \mathrm{P}=0.05 \\
\text { BKN-vastus: ns } \\
\text { PGE2-vastus: PMR >HC; } \mathrm{P}=0.05 \\
\text { K+-vastus: ns } \\
\text { ATP-vastus: ns }\end{array}$ & $\begin{array}{l}\text { Compensated } \\
\text { for RR: } Y \\
\text { Low flow: NA }\end{array}$ \\
\hline $\begin{array}{l}\text { Kreiner et } \\
\text { al. } \\
\text { (Kreiner et } \\
\text { al., 2010) }\end{array}$ & 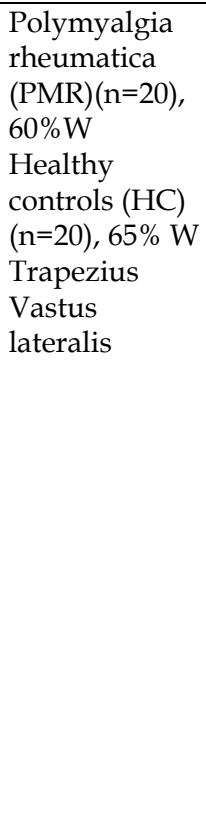 & $\begin{array}{l}\text { IL-1a/ } \beta \\
\text { IL-1 receptor } \\
\text { antagonist } \\
\text { (IL-1Ra) } \\
\text { IL-6 } \\
\text { IL-8 } \\
\text { TNF-a } \\
\text { Monocyte } \\
\text { chemoattract } \\
\text { ant } \\
\text { protein } \\
1(\mathrm{MCP}-1) \\
3 \mu \mathrm{l} / \mathrm{min}\end{array}$ & 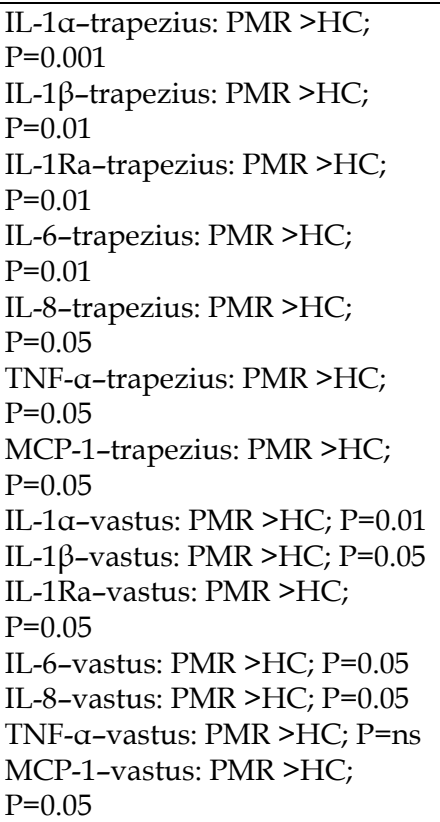 & $\begin{array}{l}\text { Compensated } \\
\text { for RR: } Y \\
\text { Low flow: NA }\end{array}$ \\
\hline
\end{tabular}

*Low flow rate associated with $100 \%$ relative recovery was defined as $0.3 \mu \mathrm{l} / \mathrm{min}$.

Table 6. Studies of Polymyalgia rheumatica. The results of the different substances are baseline data. Healthy controls are abbreviated as HC. 


\begin{tabular}{|c|c|c|c|c|}
\hline Study & \begin{tabular}{|l} 
Condition \\
Number of \\
Subjects, \% \\
women(W) \\
Muscles \\
\end{tabular} & $\begin{array}{l}\text { Substances } \\
\text { investigated } \\
\text { Flow rate }\end{array}$ & $\begin{array}{l}\text { Results } \\
\text { (Comparisons } \\
\text { between patients and } \\
\text { HC; p-values) }\end{array}$ & $\begin{array}{l}\text { Comments } \\
\text { (Compensated } \\
\text { for RR }(\mathrm{Y} / \mathrm{N}) \text { or } \\
\text { low flow rate* } \\
(\mathrm{NA} \text { or } \mathrm{Y} / \mathrm{N}) \\
\end{array}$ \\
\hline $\begin{array}{l}\text { Axelson } \\
\text { et al } \\
\text { (Axelson et } \\
\text { al., 2002) }\end{array}$ & $\begin{array}{l}\text { Mitochondrial } \\
\text { Myopathy (MM; } \\
\mathrm{n}=4) ; 50 \% \mathrm{~W} \\
\mathrm{HC}(\mathrm{n}=11), 36 \% \\
\text { W } \\
\text { Tibialis anterior }\end{array}$ & $\begin{array}{l}\text { Lactate } \\
\text { Pyruvate } \\
\text { Glutamate } \\
\text { Hypoxanthine } \\
\text { Urate } \\
\text { Aspartate } \\
0.3 \mu 1 / \mathrm{min}\end{array}$ & $\begin{array}{l}\text { Lactate-tibialis: ns } \\
\text { Pyruvate-tibialis: ns } \\
\text { Glutamate-tibialis: ns } \\
\text { Hypoxanthine- } \\
\text { tibialis: ns } \\
\text { Urate-tibialis: ns } \\
\text { Aspartate-tibialis: ns }\end{array}$ & $\begin{array}{l}\text { Compensated for } \\
\text { RR: } \mathrm{N} \\
\text { Low flow: } \mathrm{Y}\end{array}$ \\
\hline
\end{tabular}

* Low flow rate associated with $100 \%$ relative recovery was defined as $0.3 \mu \mathrm{l} / \mathrm{min}$.

Table 7. Studies of mitochondrial myopathy. The results of the different substances are baseline data. Healthy controls are abbreviated as HC.

\begin{tabular}{|c|c|c|c|c|}
\hline Study & $\begin{array}{l}\text { Condition } \\
\text { Number of } \\
\text { Subjects, \% } \\
\text { women(W) } \\
\text { Tendon }\end{array}$ & $\begin{array}{l}\text { Substances } \\
\text { investigated }\end{array}$ & $\begin{array}{l}\text { Results } \\
\text { (Comparisons between } \\
\text { patients and HC; } \\
\text { p-values) }\end{array}$ & $\begin{array}{l}\text { Comments } \\
\text { (Compensated for } \\
\text { RR }(Y / N) \text { or low } \\
\text { flow* } \\
(N A \text { or } Y / N))\end{array}$ \\
\hline $\begin{array}{l}\text { Alfredson } \\
\text { et al } \\
\text { (Alfredson et } \\
\text { al., 2000) }\end{array}$ & $\begin{array}{l}\text { Tennis Elbow } \\
(\mathrm{TE} ; \mathrm{n}=4) \text {, } \\
25 \% \mathrm{~W} \\
\mathrm{HC}(\mathrm{n}=4) \text {, } \\
50 \% \mathrm{~W} \\
\text { Extensor carpi } \\
\text { radialis brevis } \\
\text { tendon }\end{array}$ & $\begin{array}{l}\text { Glutamate } \\
\text { PGE }_{2}\end{array}$ & $\begin{array}{l}\text { Glutamate: ns } \\
\text { PGE }_{2}: \text { TE }>H C ; P<0.001\end{array}$ & $\begin{array}{l}\text { Compensated } \\
\text { for RR: } N \\
\text { Low flow: Y }\end{array}$ \\
\hline $\begin{array}{l}\text { Alfredson } \\
\text { et al } \\
\text { (Alfredson et } \\
\text { al., 2001) }\end{array}$ & $\begin{array}{l}\text { Jumpers' knee } \\
(\mathrm{JK} ; \mathrm{n}=5), \\
20 \% \mathrm{~W} \\
\mathrm{HC}(\mathrm{n}=5), \\
20 \% \mathrm{~W} \\
\text { Patellar tendon }\end{array}$ & $\begin{array}{l}\text { Glutamate } \\
\text { PGE }_{2}\end{array}$ & $\begin{array}{l}\text { Glutamate: } \mathrm{JK}>\mathrm{HC} \text {; } \\
\mathrm{P}=0.01 \\
\mathrm{PGE}_{2}: \text { ns }\end{array}$ & $\begin{array}{l}\text { Compensated } \\
\text { for RR: } \mathrm{N} \\
\text { Low flow: Y }\end{array}$ \\
\hline $\begin{array}{l}\text { Alfredson } \\
\text { et al } \\
\text { (Alfredson, } \\
2005 \text { ) }\end{array}$ & $\begin{array}{l}\text { Chronic } \\
\text { Achilles } \\
\text { tendinosis } \\
(\mathrm{CAT} ; \mathrm{n}=4), \\
0 \% \mathrm{~W} \\
\mathrm{HC}(\mathrm{n}=5), 0 \% \mathrm{~W} \\
\text { Achilles tendon }\end{array}$ & Lactate & $\begin{array}{l}\text { Lactate: } \mathrm{CAT}>\mathrm{HC} ; \\
\mathrm{P}=0.05\end{array}$ & $\begin{array}{l}\text { Compensated } \\
\text { for RR: } \mathrm{N} \\
\text { Low flow: Y }\end{array}$ \\
\hline
\end{tabular}

*Low flow was defined as $0.3 \mu \mathrm{l} / \mathrm{min}$.

Table 8. Studies of tendons with chronic pain. The results of the different substances are baseline data. Healthy controls are abbreviated as HC. 


\section{Discussion}

\subsection{Metabolites}

The majority of studies concerning the trapezius in chronic trapezius myalgia have reported increases in the interstitial concentrations of lactate and pyruvate (Flodgren et al., 2010; Flodgren et al., 2006; Larsson et al., 2008; Rosendal et al., 2004b; Sjogaard et al., 2010). However, Flodgren et al. (Flodgren et al., 2010; Flodgren et al., 2006) did not compensate for RR and their results might be biased.

There are several possible explanations for the generally higher interstitial levels of pyruvate levels in chronic trapezius myalgia and in the trapezius of patients with FMS. For example, changes in the lactate-pyruvate metabolism via lactate dehydrogenase isoforms may result in higher pyruvate levels (Philip et al., 2005). Another explanation is a reduction in tissue oxygenation in FMS (Bengtsson, 2002) and chronic trapezius myalgia (Larsson et al., 2004), reductions that may result in higher pyruvate and higher lactate concentrations due to a shift towards an anaerobic state. A lower fitness level is a third explanation as a low fitness level means more frequent reliance on anaerobic metabolism. However, it is unknown if a general deconditioning in these two pain conditions involves the postural trapezius. The aerobic capacity of a muscle is largely governed by the number of mitochondria and their enzymes (Weibel \& Hoppeler, 2005). The mitochondrial density increases as result of exercise and this increased density affects the level of metabolites (i.e., enhanced aerobic capacity) (Norrbom, 2008). Lower capillary density and/or enzymes associated with aerobic metabolism have been reported in FMS and in chronic trapezius myalgia (Larsson et al., 2004; Lindh et al., 1995). For FMS and chronic trapezius myalgia, the trapezius muscle fibres can appear with alterations in mitochondrial content and distribution, e.g., moth-eaten fibres and ragged red-fibres (Bengtsson, 2002; Bengtsson et al., 1986; Larsson et al., 2000; Larsson et al., 2004).

The role of lactate is complex. Lactate may assist in the detection of exercise stress before tissue damage occurs and can be exchanged rapidly among tissue compartments where it may be oxidized as a fuel or reconverted to form pyruvate or glucose (Gladden, 2004; Kim et al., 2007; Philip et al., 2005; Robergs et al., 2004). Lactate is also involved in peripheral nociception and it appears to facilitate the response of the acid-sensing ion channel 3 (ASIC3) to low pH (Kim et al., 2007). Such ASIC channels are considered to be molecular transducers for nociception and mechanosensation (Kim et al., 2007).

To summarize, most studies of myalgic trapezius muscles show significant increases in interstitial levels of lactate and pyruvate. These results might be explained by decreased fitness level, reduced tissue oxygenation, increased muscle activation, and/or damaged mitochondria.

\subsection{Pain-related substances}

\subsubsection{Glutamate}

Two of the studies of chronic trapezius myalgia (Larsson et al., 2008; Rosendal et al., 2004b), which are markedly larger than the third study (Flodgren et al., 2005), found significant increases in the interstitial concentrations of glutamate. A possible difference between subjects of these studies may contribute to the inconsistent glutamate finding. The myalgic 
subjects studied by Larsson et al. and Rosendal et al. (Larsson et al., 2008; Rosendal et al., 2004b) comprised subjects reporting considerable pain and had distinct current muscular signs confirmed at clinical examination. The pain history, the present pain, and clinical muscular neck status of the subjects are very sparsely presented in the Flodgren study (Flodgren et al., 2005). Moreover, one study found the painful masseter was significantly associated with increased glutamate (Castrillon et al., 2010).

Glutamate, a pain modulator in the human central nervous system, acts via the N-methyl-Daspartate (NMDA) receptor (Coggeshall \& Carlton, 1997) (Hudspith, 1997) and influences peripheral pain processing (Carlton, 2001; Varney \& Gereau, 2002 ), e.g., muscle inflammation and delayed onset muscle soreness (Cairns et al., 2001a; Cairns et al., 2001b; Cairns et al., 2003; Svensson et al., 2003; Svensson et al., 2005; Tegeder et al., 2002 ). Glutamate is released from peripheral afferent nerve terminals (Miller et al., 2011). Studies of animals have shown that glutamate receptors are located on the peripheral ends of smalldiameter primary afferents in several tissues such as muscle (Coggeshall \& Carlton, 1998). Inflammatory animal models reveal increased levels of glutamate in peripheral tissues and nociceptive behaviours (Miller et al., 2011). Several studies have demonstrated that injections of glutamate increase pain intensity (Cairns et al., 2003; Gazerani et al., 2006). A review from 2008 concluded that elevation of interstitial glutamate in skeletal muscle alters pain sensitivity in healthy humans and is associated with pain symptoms in some chronic non-inflammatory muscle pain conditions (Cairns \& Dong, 2008), which are probably mediated through activation of peripheral excitatory amino acid receptors located on the terminal ends of nociceptors. The present review mainly supports the conclusions of that review. However, the interstitial concentrations of glutamate were not increased in the trapezius of patients with chronic WAD (Gerdle et al., 2008c) or in patients with fibromyalgia (Gerdle et al., 2010). One difference between chronic trapezius myalgia and the two other conditions might be the more widespread (spatial) hyperalgesia in the two latter conditions (Arendt-Nielsen \& Graven-Nielsen, 2003; Wallin et al., 2011).

\subsubsection{Serotonin}

Serotonin (5-hydroxtryptamine, 5-HT) is involved in the central and peripheral modulation of nociceptive pain and hyperalgesia (Sommer, 2004). 5-HT is synthesized in brain neurons from the essential amino acid tryptophan and released from platelets and mast cells in the periphery due to tissue damage (Mense, 1993). Whether 5-HT has an analgesic or hyperalgesic action depends on the cell type and type of receptor it targets. Currently, seven receptor families have been identified. These receptors are cell-surface proteins that bind 5HT and trigger intracellular changes that influence the behaviour of cells and explain the broad physiological actions and distribution of this biochemical mediator. According to animal studies, the 5-HT receptor classes currently identified, the 5- $\mathrm{HT}_{1}$ and the 5- $\mathrm{HT}_{2}$, may be involved in mainly chemical and thermal hyperalgesia (Ernberg, 2008). 5-HT activates the descending endogenous pain system and this inhibits centrally mediated pain transduction (Sommer, 2006; Suzuki et al., 2004). In the periphery, 5-HT sensitizes afferent nerve fibres, contributing to hyperalgesia in inflammation and nerve injury (Giordano \& Rogers, 1989; Sommer, 2004; Taiwo \& Levine, 1992). Intramuscular administration of 5-HT into the human masseter muscle has been demonstrated to induce pain (Ernberg et al., 2006). The studies identified in this systematic review concerning chronic trapezius myalgia (Table 1) clearly 
indicate significantly increased levels of 5-HT in the whole spectrum of severity of chronic trapezius myalgia and these findings agree with other studies of muscle pain conditions such as myalgic masseter muscle (Ernberg et al., 1999) (Table 4) and chronic whiplash associated pain including trapezius pain (Gerdle et al., 2008c) (Table 2). The finding that there are higher interstitial levels of 5-HT in chronic trapezius myalgia than in controls at rest agrees with other studies that also found that 5-HT is a peripheral pro-nociceptive substance activated by afferents and by the release of other substances (Saria et al., 1990; Sommer, 2004). The results of the present systematic review clearly indicate that 5-HT can be a potential biomarker of different types and severity of chronic myalgia.

\subsubsection{Bradykinin (BKN) and Kallidin (KAL)}

BKN and KAL are kinins - a group of structurally related 9-11 amino acid peptides that are produced by kallikrein-mediated enzymatic cleavage of kininogen (Coutaux et al., 2005; Riedel \& Neeck, 2001; Wang et al., 2006). Kinins mediate their effects via two different G protein coupled receptors, $\mathrm{B}_{1}$ and $\mathrm{B}_{2}$, that provoke an increase in intracellular $\mathrm{Ca}^{2+}$ (Meyer et al., 2006; Zubakova et al., 2008). In normal tissue in the acute situation, BKN and KAL act via the $B_{2}$ receptor. In the chronic phase of the response of tissue injury and infection, $B_{1}$ receptors are expressed by BKN and KAL via this receptor (Calixto et al., 2004; Coutaux et al., 2005; Couture et al., 2001; Graven-Nielsen \& Mense, 2001; McMahon et al., 2006). Interstitial muscle BKN and KAL have been suggested as algesic kinins involved in muscle pain. BKN was the first inflammatory mediator recognized to have potent hyperalgesic properties (Levine \& Reichling, 1999). BKN induces pain and modifies the receptive fields of dorsal horn neurons to noxious stimuli in humans when administered in different ways (Boix et al., 2005; Meyer et al., 2006). BKN and cytokines are central factors in the link between tissue damage and inflammatory responses (Coutaux et al., 2005). Moreover, BKN is a potent vasodilatator and is increased in the interstitium of muscle during exercise (Clifford \& Hellsten, 2004; Schmelz et al., 2003; Stewart \& Rittweger, 2006). Animal studies have shown that BKN can both excite (i.e., algogenic) and sensitize nociceptors (Levine \& Reichling, 1999; Wang et al., 2006). The present review identified four studies investigating the interstitial concentrations of BKN and/or Kallidin (Gerdle et al., 2008b; Larsson et al., 2008; Shah et al., 2008; Shah et al., 2005). The relatively small studies conducted by Shah et al. (Shah et al., 2008; Shah et al., 2005) clearly indicated that BKN was involved since increased levels of $\mathrm{BKN}$ in subjects with active trigger points and the levels were higher in the trapezius (with pain) than in a pain-free distant muscle. In contrast no significant differences in BKN were found between patients and controls in a field study and a laboratory study, (Gerdle et al., 2008b; Larsson et al., 2008) The difference in results between the above mentioned studies could be due to the fact that alterations in BKN might be very localized (i.e., in the trigger points) and not generally found in the aching trapezius muscle. KAL was only investigated in one study and increased in chronic trapezius myalgia but not in the trapezius of chronic WAD compared to controls (Gerdle et al., 2008b). Clearly, more pathophysiological in vivo studies are necessary in order to understand the roles of BKN and KAL for nociception and pain in patients with chronic pain.

\subsubsection{Potassium}

Increased interstitial potassium levels may be related to muscle pain (Graven-Nielsen et al., 1997). Green et al., however, did not find potassium related to acute ischaemic myalgia in 
healthy subjects (Green et al., 2000). Repetitive work in healthy subjects may increase potassium levels (Rosendal et al., 2004a), although in the present systematic review no consistent pattern of increased potassium was found in patients with chronic trapezius myalgia (Larsson et al., 2008; Rosendal et al., 2005; Sjogaard et al., 2010) (Table 1) or with chronic WAD (Table 2).

\subsubsection{Cytokines}

There are several direct and indirect pathways that link cytokines with nociception or hyperalgesia (Coutaux et al., 2005; Sommer \& Kress, 2004; Uceyler et al., 2009). Four studies investigated cytokines in chronic trapezius myalgia, but significant differences were only found for active trigger points (Shah et al., 2008; Shah et al., 2005). These studies, however, used very few subjects, an obvious limitation. Larger studies have not found elevated levels of cytokines (Larsson et al., 2008; Rosendal et al., 2005). On the other hand, these studies might have had some technical problems due to the catheters used, also a limitation. However, the chronic WAD study found increased IL-6 (Gerdle et al., 2008c).

\subsection{Anti-analgesic substances}

As is obvious from the above, most studies concern metabolites and algesic substances. Little is known about changes in the pain-inhibitory signalling molecules. One interesting group of such molecules is the $N$-acylethanolamines (NAEs), which is a family of endogenous lipid mediators that have several roles including the regulation of inflammation and pain (Pacher et al., 2006). Examples of NAEs are N-palmitoylethanolamine (PEA), Nstearoylethanolamine (SEA), N-oleoylethanolamine (OEA), and N-arachidonoylethanolamine (anandamide, AEA). The most thoroughly studied of the NAEs is AEA, which interacts with cannabinoid receptors. At higher concentrations, AEA also targets transient receptor potential (vanilloid-1) receptors and has been shown to have anti-nociceptive actions in a number of animal models of pain (Calignano et al., 1998). The present review also includes one study of SEA and PEA (Ghafouri et al., 2011), which reported significantly increased levels of two NAEs.

\subsection{Other pain conditions identified}

According to our review, microdialysis of painful muscles have also been investigated, but these studies have only been based on one group of patients of each condition: polymyalgia rheumatic, chronic tension-type headache, and mitochondrial myopathy. Hence no definite conclusions concerning potential biomarkers can be drawn for these conditions. The results concerning polymyalgia rheumatic were prominent for several algesics including cytokines. Kreiner et al. investigated the presence of muscle alterations (Kreiner \& Galbo, 2011; Kreiner et al., 2010), and they found that the biochemical alterations were normalized after treatment with prednisolone. Although more studies are needed, their results suggest that intramuscular mechanisms are important.

In the studies of different chronic tendinosis, the number of patients was low and no consistent patterns were seen. Larger studies are needed to identify biochemical alterations. 


\subsection{Suggestions with respect to future studies}

The fact that most studies of chronic trapezius myalgia, chronic WAD, and temporomandibular pain disorders included women is expected, as the prevalence of these conditions are higher in women. Future studies should also include groups of men with chronic muscle pain conditions. In addition, it is important to describe the patient group in detail with respect to clinically relevant examination parameters. Furthermore, systematic descriptions of the patient groups are needed that reflect pain intensity and psychological distress as well as consequences such as work participation and sick leave. A systematic description will allow a more accurate characterization of pain severity in a broad context. Most studies rely on bivariate correlations between pain descriptors such as pain intensity or pressure pain thresholds and the concentration of a certain substance. Multivariate correlation analyses and regression analyses are methods that can be used to investigate how groups of clinical examination variables, several simultaneous symptoms, and the concentrations of several biochemical substances intercorrelate. To better understand the potentially complex biochemical situation of the muscle in chronic pain conditions, it is necessary to also investigate the multivariate interrelationships between the concentrations of the investigated substances. According to ICD, the clinically used pain diagnoses are symptom diagnoses based on temporal and anatomical characteristics (e.g., chronic lumbago). Hence a certain diagnosis may include patients with different activated pathophysiological mechanisms. To identify subgroups of patients with identical pathophysiological mechanisms, it is important to use large patient groups and appropriate statistical methods (e.g., cluster analysis and principal component analysis).

\section{Conclusion}

This systematic review found that most of the studies focused on trapezius myalgia (seven patient groups reported in ten studies), temporomanidibular pain syndromes (two patient groups reported in three studies), and fibromyalgia (two patient groups but different muscles). Relatively strong scientific support identifies 5-HT as a potential biomarker in chronic myalgia. Moderately strong scientific support identifies glutamate, pyruvate, and lactate as potential biomarkers in chronic trapezius myalgia. There is a need for larger studies of well-characterized patient groups with respect to perceived situations, symptoms, and signs so as to investigate several substances simultaneously in order to improve the understanding of peripheral nociceptive processes in myalgia.

\section{Acknowledgements}

There are no conflicts of interest. No financial or personal relationships have inappropriately influenced this work. This study was supported by the Swedish Council for Working Life and Social Research (2010-0683, 2007-0760) and the Swedish Research Council (2010-2893). The funders had no role in study design, data collection and analysis, decision to publish, or preparation of the manuscript.

\section{References}

Afinowi, R., Tisdall, M., Keir, G., Smith, M., Kitchen, N., \& Petzold, A. (2009). Improving the recovery of S100B protein in cerebral microdialysis: implications for multimodal monitoring in neurocritical care. Journal of neuroscience methods, 181, pp. 95-99. 
Agarwal, N., Pacher, P., Tegeder, I., Amaya, F., Constantin, CE., Brenner, GJ., Rubino, T., Michalski, CW., Marsicano, G., Monory, K., Mackie, K., Marian, C., Batkai, S., Parolaro, D., Fischer, MJ., Reeh, P., Kunos, G., Kress, M., Lutz, B., Woolf, CJ., \& Kuner, R. (2007). Cannabinoids mediate analgesia largely via peripheral type 1 cannabinoid receptors in nociceptors. Nat Neurosci, 10, pp. 870-879.

Alfredson, H. (2005). The chronic painful Achilles and patellar tendon: research on basic biology and treatment. Scandinavian journal of medicine $\mathcal{E}$ science in sports, 15, pp. 252-259.

Alfredson, H., Forsgren, S., Thorsen, K., \& Lorentzon, R. (2001). In vivo microdialysis and immunohistochemical analyses of tendon tissue demonstrated high amounts of free glutamate and glutamate NMDAR1 receptors, but no signs of inflammation, in Jumper's knee. J Orthop Res, 19, pp. 881-886.

Alfredson, H., Ljung, BO., Thorsen, K., \& Lorentzon, R. (2000). In vivo investigation of ECRB tendons with microdialysis technique--no signs of inflammation but high amounts of glutamate in tennis elbow. Acta Orthop Scand, 71, pp. 475-479.

Alonso, J., Angermeyer, MC., Bernert, S., Bruffaerts, R., Brugha, TS., Bryson, H., de Girolamo, G., Graaf, R., Demyttenaere, K., Gasquet, I., Haro, JM., Katz, SJ., Kessler, RC., Kovess, V., Lepine, JP., Ormel, J., Polidori, G., Russo, LJ., Vilagut, G., Almansa, J., Arbabzadeh-Bouchez, S., Autonell, J., Bernal, M., Buist-Bouwman, MA., Codony, M., Domingo-Salvany, A., Ferrer, M., Joo, SS., Martinez-Alonso, M., Matschinger, H., Mazzi, F., Morgan, Z., Morosini, P., Palacin, C., Romera, B., Taub, N., \& Vollebergh, WA. (2004). 12-Month comorbidity patterns and associated factors in Europe: results from the European Study of the Epidemiology of Mental Disorders (ESEMeD) project. Acta Psychiatr Scand Suppl, pp. 28-37.

Apkarian, AV. (2008). Pain perception in relation to emotional learning. Curr Opin Neurobiol, 18, pp. 464-468.

Arendt-Nielsen, L., \& Graven-Nielsen, T. (2003). Central sensitization in fibromyalgia and other musculoskeletal disorders. Current Pain and Headache Reports, 7, pp. 355-361.

Ashina, M., Stallknecht, B., Bendtsen, L., Pedersen, JF., Galbo, H., Dalgaard, P., \& Olesen, J. (2002). In vivo evidence of altered skeletal muscle blood flow in chronic tensiontype headache. Brain, 125, pp. 320-326.

Ashina, M., Stallknecht, B., Bendtsen, L., Pedersen, JF., Schifter, S., Galbo, H., \& Olesen, J. (2003). Tender points are not sites of ongoing inflammation -in vivo evidence in patients with chronic tension-type headache. Cephalalgia, 23, pp. 109-116.

Asmundson, GJG., \& Katz, J. (2009). Understanding the co-occurrence of anxiety disorders and chronic pain: state-of-the-art. Depress Anxiety, 26, pp. 888-901.

Axelson, HW., Melberg, A., Ronquist, G., \& Askmark, H. (2002). Microdialysis and electromyography of experimental muscle fatigue in healthy volunteers and patients with mitochondrial myopathy. Muscle Nerve, 26, pp. 520-526.

Baliki, M., Schnitzer, T., Bauer, W., \& Apkarian, A. (2011). Brain morphological Signatures for chronic Pain. PLoS One, 6, pp. e26010.

Bengtsson, A. (2002). Editorial, The muscle in fibromyalgia. Rheumatol 41, pp. 721-724.

Bengtsson, A., Henriksson, KG., \& Larsson, J. (1986). Muscle biopsy in primary fibromyalgia. Light-microscopical and histochemical findings. Scand J Rheumatol, 15, pp. 1-6. 
Bernard, BP. Musculosceletal disorders and workplace factors. In: A critical review of epidemiological evidence for work-related musculosceletal disorders of the neck, upper extremity, and low back.NIOSH: DHHS; 1997.

Boix, F., Roe, C., Rosenborg, L., \& Knardahl, S. (2005). Kinin peptides in human trapezius muscle during sustained isometric contraction and their relation to pain. Journal of applied physiology, 98, pp. 534-540.

Breivik, H., Collett, B., Ventafridda, V., Cohen, R., \& Gallacher, D. (2006). Survey of chronic pain in Europe: prevalence, impact on daily life, and treatment. Eur J Pain, 10, pp. 287-333.

Börsbo, B., Peolsson, M., \& Gerdle, B. (2008). Catastrophizing, depression, and pain: Correlation with and influence on quality of life and health - A study of chronic whiplash-associated disorders. J Rehabil Med, 40, pp. 562-569.

Cairns, BE., \& Dong, X. (2008). The role of peripheral glutamate and glutamate receptors in muscle pain. J Musculoskelet Pain, 16, pp. 85-91.

Cairns, BE., Hu, JW., Arendt-Nielsen, L., Sessle, BJ., \& Svensson, P. (2001a). Sex-related differences in human pain and rat afferent discharge evoked by injection of glutamate into the masseter muscle. J Neurophysiol, 86, pp. 782-789.

Cairns, BE., Sessle, BJ., \& Hu, JW. (2001b). Characteristics of glutamate-evoked temporomandibular joint afferent activity in the rat. J Neurophysiol, 85, pp. 24462445 .

Cairns, BE., Wang, K., Hu, JW., Sessle, BJ., Arendt-Nielsen, L., \& Svensson, P. (2003). The effect of glutamate-evoked masseter muscle pain on the human jaw-stretch reflex differs in men and women. Journal of orofacial pain, 17, pp. 317-325.

Calignano, A., La Rana, G., Giuffrida, A., \& Piomelli, D. (1998). Control of pain initiation by endogenous cannabinoids. Nature 394, pp. 277-281.

Calixto, JB., Medeiros, R., Fernandes, ES., Ferreira, J., Cabrini, DA., \& Campos, MM. (2004). Kinin B1 receptors: key G-protein-coupled receptors and their role in inflammatory and painful processes. Br J Pharmacol, 143, pp. 803-818.

Carlton, SM. (2001). Peripheral excitatory amino acids. Curr Opin Pharmacol, 1, pp. 52-56.

Castrillon, EE., Ernberg, M., Cairns, BE., Wang, K., Sessle, BJ., Arendt-Nielsen, L., \& Svensson, P. (2010). Interstitial glutamate concentration is elevated in the masseter muscle of myofascial temporomandibular disorder patients. J Orofac Pain, 24, pp. 350-360.

Clifford, PS., \& Hellsten, Y. (2004). Vasodilatory mechanisms in contracting skeletal muscle. J Appl Physiol, 97, pp. 393-403.

Coggeshall, RE., \& Carlton, SM. (1997). Receptor localization in the mammalian dorsal horn and primary afferent neurons. Brain research Brain research reviews, 24, pp. 28-66.

Coggeshall, RE., \& Carlton, SM. (1998). Ultrastructural analysis of NMDA, AMPA, and kainate receptors on unmyelinated and myelinated axons in the periphery. J Comp Neurol, 391, pp. 78-86.

Coutaux, A., Adam, F., Willer, JC., \& Le Bars, D. (2005). Hyperalgesia and allodynia: peripheral mechanisms. Joint Bone Spine, 72, pp. 359-371.

Couture, R., Harrisson, M., Vianna, RM., \& Cloutier, F. (2001). Kinin receptors in pain and inflammation. Eur J Pharmacol, 429, pp. 161-176.

Dahlin, A., Wetterhall, M., Caldwell, K., Larsson, A., Bergquist, J., Hillered, L., \& Hjort, K. $(2010$ ). Methodological aspects on microdialysis protein sampling and 
quantification in biological fluids: an in vitro study on human ventricular CSF. Anal Chem, 82, pp. 4376-4385.

Dersh, J., Gatchel, RJ., \& Polatin, P. (2001). Chronic spinal disorders and psychopathology: research findings and theoretical considerations. The Spine Journal, 1, pp. 88-94.

Ericsson, M., Poston, W., Linder, J., Taylor, J., Haddock, C., \& Foreyt, J. ( 2002). Depression predicts disability in long-term chronic pain patients. Disabil Rehabil, 24, pp. 334340.

Ernberg, M. Serotonergic Receptor involvement in Muscle pain and hyperalgesia. In: Fundamentals of Musculoskeltal Pain. 1th. Seattle: IASP Press; 2008; 139-153.

Ernberg, M., Hedenberg-Magnusson, B., Alstergren, P., \& Kopp, S. (1999). The level of serotonin in the superficial masseter muscle in relation to local pain and allodynia. Life sciences, 65, pp. 313-325.

Ernberg, M., Hedenberg-Magnusson, B., Kurita, H., \& Kopp, S. (2006). Effects of local serotonin administration on pain and microcirculation in the human masseter muscle. J Orofac Pain, 20, pp. 241-248.

Flodgren, GM., Crenshaw, AG., Alfredson, H., Fahlstrom, M., Hellstrom, FB., Bronemo, L., \& Djupsjobacka, M. (2005). Glutamate and prostaglandin E2 in the trapezius muscle of female subjects with chronic muscle pain and controls determined by microdialysis. European journal of pain, 9, pp. 511-515.

Flodgren, GM., Crenshaw, AG., Hellstrom, F., \& Fahlstrom, M. (2010). Combining microdialysis and near-infrared spectroscopy for studying effects of low-load repetitive work on the intramuscular chemistry in trapezius myalgia. J Biomed Biotechnol, 2010, pp. 513803.

Flodgren, GM., Hellstrom, FB., Fahlstrom, M., \& Crenshaw, AG. (2006). Effects of 30 versus $60 \mathrm{~min}$ of low-load work on intramuscular lactate, pyruvate, glutamate, prostaglandin $\mathrm{E}(2)$ and oxygenation in the trapezius muscle of healthy females. European journal of applied physiology, 97, pp. 557-565.

Gatchel, R., Peng, Y., Peters, M., Fuchs, P., \& Turk, D. (2007). The biopsychosocial approach to chronic pain: scientific advances and future directions. Psychol Bull, 133, pp. 581624.

Gazerani, P., Wang, K., Cairns, BE., Svensson, P., \& Arendt-Nielsen, L. (2006). Effects of subcutaneous administration of glutamate on pain, sensitization and vasomotor responses in healthy men and women. Pain, 124, pp. 338-348.

Gerdle, B., Bjork, J., Coster, L., Henriksson, KG., Henriksson, C., \& Bengtsson, A. (2008a). Prevalence of widespread pain and associations with work status: a population study. Bmc Musculoskeletal Disorders, 9, pp.

Gerdle, B., Bjork, J., Henriksson, C., \& Bengtsson, A. (2004). Prevalence of current and chronic pain and their influences upon work and healthcare-seeking: A population study. Journal of Rheumatology, 31, pp. 1399-1406.

Gerdle, B., Hilgenfeldt, U., Larsson, B., Kristiansen, J., Sogaard, K., \& Rosendal, L. (2008b). Bradykinin and kallidin levels in the trapezius muscle in patients with workrelated trapezius myalgia, in patients with whiplash associated pain, and in healthy controls - A microdialysis study of women. Pain, 139, pp. 578-587.

Gerdle, B., Lemming, D., Kristiansen, J., Larsson, B., Peolsson, M., \& Rosendal, L. (2008c). Biochemical alterations in the trapezius muscle of patients with chronic whiplash 
associated disorders (WAD) - A microdialysis study. European Journal of Pain, 12, pp. 82-93.

Gerdle, B., Soderberg, K., Salvador Puigvert, L., Rosendal, L., \& Larsson, B. (2010). Increased interstitial concentrations of pyruvate and lactate in the trapezius muscle of patients with fibromyalgia: a microdialysis study. J Rehabil Med, 42, pp. 679-687.

Ghafouri, B., Larsson, BK., Sjors, A., Leandersson, P., \& Gerdle, BU. (2010). Interstitial concentration of serotonin is increased in myalgic human trapezius muscle during rest, repetitive work and mental stress - an in vivo microdialysis study. Scand J Clin Lab Invest, 70, pp. 478-486.

Ghafouri, N., Ghafouri, B., Larsson, B., Turkina, M., Karlsson, L., Fowler, C., \& Gerdle, B. (2011). High levels of N-palmitoylethanolamide and N-stearoylethanolamide in microdialysate samples from myalgic trapezius muscle in women. PLOS One, 6 (11), pp e27257; doi:10.1371/journal.pone.0027257.

Giordano, J., \& Rogers, LV. (1989). Peripherally administered serotonin 5-HT3 receptor antagonists reduce inflammatory pain in rats. European journal of pharmacology, 170, pp. 83-86.

Gladden, LB. (2004). Lactate metabolism: a new paradigm for the third millenium. J Physiol (Lond), 558, pp. 5-30.

Grachev, ID., Fredrickson, BE., \& Apkarian, AV. (2000). Abnormal brain chemistry in chronic back pain: an in vivo proton magnetic resonance spectroscopy study. Pain, 89, pp. 7-18.

Graven-Nielsen, T., McArdle, A., Phoenix, J., Arendt-Nielsen, L., Jensen, TS., Jackson, MJ., \& Edwards, RH. (1997). In vivo model of muscle pain: quantification of intramuscular chemical, electrical, and pressure changes associated with saline-induced muscle pain in humans. Pain, 69, pp. 137-143.

Graven-Nielsen, T., \& Mense, S. (2001). The peripheral apparatus of muscle pain: evidence from animal and human studies. Clin J Pain, 17, pp. 2-10.

Green, S., Langberg, H., Skovgaard, D., Bulow, J., \& Kjaer, M. (2000). Interstitial and arterialvenous $[\mathrm{K}+]$ in human calf muscle during dynamic exercise: effect of ischaemia and relation to muscle pain. The Journal of physiology, $529 \mathrm{Pt} 3$, pp. 849-861.

Guez, M., Hildingsson, C., Nilsson, M., Toolanen, G., \& Aug;():. (2002). The prevalence of neck pain: a population-based study from northern Sweden. Acta Orthop Scand, 73, pp. 455-459.

Hamrin, K., Rosdah, 1H., Ungerstedt, U., \& Henriksson, J. (2002 ). Microdialysis in human skeletal muscle: effects of adding a colloid to the perfusate. J Appl Physiol, 92, pp. 385-393.

Hedenberg-Magnusson, B., Ernberg, M., Alstergren, P., \& Kopp, S. (2001). Pain mediation by prostaglandin E2 and leukotriene B4 in the human masseter muscle. Acta odontologica Scandinavica, 59, pp. 348-355.

Heinricher, MM., Tavares, I., Leith, JL., \& Lumb, BM. (2009). Descending control of nociception: Specificity, recruitment and plasticity. Brain Res Rev, 60, pp. 214-225.

Helmy, A., Carpenter, KL., Skepper, JN., Kirkpatrick, PJ., Pickard, JD., \& Hutchinson, PJ. (2009). Microdialysis of cytokines: methodological considerations, scanning electron microscopy, and determination of relative recovery. J Neurotrauma, 26, pp. 549-561. 
Hudspith, MJ. (1997). Glutamate: a role in normal brain function, anaesthesia, analgesia and CNS injury. Br J Anaesth, 78, pp. 731-747.

Hunt, SP., \& Mantyh, PW. Understanding the neurobiology of chronic pain: molecular and cellular biology. In: Pain 2002- An updated Review: Refresher Course Syllabus.Seattle: IASP Press; 2002; 237-247.

Iannetti, GD., \& Mouraux, A. (2010). From the neuromatrix to the pain matrix (and back). Exp Brain Res, 205, pp. 1-12.

Julien, N., Goffaux, P., Arsenault, P., \& Marchand, S. (2005). Widespread pain in fibromyalgia is related to a deficit of endogenous pain inhibition. Pain, 114, pp. 295302.

Kim, TJ., Freml, L., Park, SS., \& Brennan, TJ. (2007). Lactate concentrations in incisions indicate ischemic-like conditions may contribute to postoperative pain. J Pain, 8, pp. 59-66.

Kreiner, F., \& Galbo, H. (2011). Elevated muscle interstitial levels of pain-inducing substances in symptomatic muscles in patients with polymyalgia rheumatica. Pain, 152, pp. 1127-1132.

Kreiner, F., Langberg, H., \& Galbo, H. (2010). Increased muscle interstitial levels of inflammatory cytokines in polymyalgia rheumatica. Arthritis Rheum, 62, pp. 37683775.

Kuner, R. (2010). Central mechanisms of pathological pain. Nat Med, 16, pp. 1258-1266.

Langley, CK., Aziz, Q., Bountra, C., Gordon, N., Hawkins, P., Jones, A., Langley, G., Nurmikko, T., \& Tracey, I. (2008). Volunteer studies in pain research--opportunities and challenges to replace animal experiments: the report and recommendations of a Focus on Alternatives workshop. Neuroimage, 42, pp. 467-473.

Larsson, B., Bjork, J., Henriksson, KG., Gerdle, B., \& Lindman, R. (2000). The prevalences of cytochrome c oxidase negative and superpositive fibres and ragged-red fibres in the trapezius muscle of female cleaners with and without myalgia and of female healthy controls. Pain, 84, pp. 379-387.

Larsson, B., Bjork, J., Kadi, F., Lindman, R., \& Gerdle, B. (2004). Blood supply and oxidative metabolism in muscle biopsies of female cleaners with and without myalgia. Clin J Pain, 20, pp. 440-446.

Larsson, B., Björk, J., Börsbo, B., \& Gerdle, B. (in press). A systematic review of risk factors associated with transitioning from regional musculoskeletal pain to chronic widespread pain. Eur J Pain, pp.

Larsson, B., Rosendal, L., Kristiansen, J., Sjogaard, G., Sogaard, K., Ghafouri, B., Abdiu, A., Kjaer, M., \& Gerdle, B. (2008). Responses of algesic and metabolic substances to $8 \mathrm{~h}$ of repetitive manual work in myalgic human trapezius muscle. Pain, 140, pp. 479490.

Larsson, B., Sogaard, K., \& Rosendal, L. (2007). Work related neck-shoulder pain: a review on magnitude, risk factors, biochemical characteristics, clinical picture and preventive interventions. Best Pract Res Clin Rheumatol, 21, pp. 447-463.

Lascelles, B., \& Flecknell, P. (2010). Do animal models tell us about human pain? Pain Clinical Updates, 18, pp. 1-6.

Lee, MC., \& Tracey, I. (2010). Unravelling the mystery of pain, suffering, and relief with brain imaging. Curr Pain Headache Rep, 14, pp. 124-131. 
Legrain, V., Iannetti, GD., Plaghki, L., \& Mouraux, A. (2011). The pain matrix reloaded: a salience detection system for the body. Prog Neurobiol, 93, pp. 111-124.

Levine, JD., \& Reichling, DB. Peripheral mechanisms of inflammatory pain. In: Textbook of Pain, 4th ed.Edinburgh: Churchill Livingstone; 1999; 59-84.

Lidgren, L. (2008). Preface: Neck pain and the decade of the Bone and Joint 2000-2010. Spine, 33, pp. S1-S2.

Lindh, M., Johansson, G., Hedberg, M., Henning, GB., \& Grimby, G. (1995). Muscle fiber characteristics, capillaries and enzymes in patients with fibromyalgia and controls. Scand J Rheumatol, 24, pp. 34-37.

Linley, JE., Rose, K., Ooi, L., \& Gamper, N. (2010). Understanding inflammatory pain: ion channels contributing to acute and chronic nociception. Pflugers Arch, 459, pp. 657669.

McIver, KL., Evans, C., Kraus, RM., Ispas, L., Sciotti, VM., \& Hickner, RC. (2006). NOmediated alterations in skeletal muscle nutritive blood flow and lactate metabolism in fibromyalgia. Pain, 120, pp. 161-169.

McMahon, SB., Bennett, DLH., \& Bevan, S. Inflammatory mediators and modulators of pain. In: Texbook of Pain, 5th ed. Elsevier; 2006; 49-72.

Means-Christensen, AJ., Roy-Byrne, PP., Sherbourne, CD., Craske, MG., \& Stein, MB. (2008). Relationships among pain, anxiety, and depression in primary care. Depression $\mathcal{E}$ Anxiety (1091-4269), 25, pp. 593-600.

Mense, S. (1993). Nociception from skeletal muscle in relation to clinical muscle pain. Pain, 54, pp. 241-289.

Mense, S. (2003). The pathogenesis of muscle pain. Current pain and headache reports, 7, pp. 419-425.

Mense, S. (2009). Algesic agents exciting muscle nociceptors. Exp Brain Res, 196, pp. 89-100.

Meyer, RA., Ringkamp, M., Campbell, JN., \& Raja, SN. Peripheral mechanisms of cutaneous nociception. In: Textbook of Pain , 5th ed. Elsevier; 2006; 3-34.

Miller, KE., Hoffman, EM., Sutharshan, M., \& Schechter, R. (2011). Glutamate pharmacology and metabolism in peripheral primary afferents: physiological and pathophysiological mechanisms. Pharmacol Ther, 130, pp. 283-309.

Momin, A., \& McNaughton, PA. (2009). Regulation of firing frequency in nociceptive neurons by pro-inflammatory mediators. Exp Brain Res, 196, pp. 45-52.

Norrbom, J.Exercise and Regulation of Mithochondrial Biogenesis Factors in Human Skeletal Muscle. Stockholm: Karolinska Institute; 2008; 61.

Ocañez, KLS., Kathryn McHugh, R., \& Otto, MW. (2010). A meta-analytic review of the association between anxiety sensitivity and pain. Depression and Anxiety, 27, pp. 760-767.

Ossipov, MH., Dussor, GO., \& Porreca, F. (2010). Central modulation of pain. J Clin Invest, 120, pp. 3779-3787.

Pace, MC., Mazzariello, L., Passavanti, MB., Sansone, P., Barbarisi, M., \& Aurilio, C. (2006). Neurobiology of pain. J Cell Physiol, 209, pp. 8-12.

Pacher, P., Batkai, S., \& Kunos, G. (2006). The endocannabinoid system as an emerging target of pharmacotherapy. Pharmacol Rev, 58, pp. 389-462.

Petersen-Felix, S., \& Curatolo, M. (2002). Neuroplasticity--an important factor in acute and chronic pain. Swiss Med Wkly, 132, pp. 273-278. 
Philip, A., Macdonald, AL., \& Watt, PW. (2005). Lactate - a signal coordnating cell and systemic function. . Exp Biol, 208, pp. 4561-4575.

Phillips, CJ. (2006). Economic burden of chronic pain. Expert Rev Pharmacoecon Outcomes Res, 6, pp. 591-601.

Plock, N., \& Kloft, C. (2005 ). Microdialysis--theoretical background and recent implementation in applied life-sciences. Eur J Pharm Sci, 25, pp. 1-24.

Porreca, F., Ossipov, MH., \& Gebhart, GF. (2002). Chronic pain and medullary descending facilitation. Trends Neurosci, 25, pp. 319-325.

Ptolemy, AS., \& Rifai, N. (2010). What is a biomarker? Research investments and lack of clinical integration necessitate a review of biomarker terminology and validation schema. Scand J Clin Lab Invest Suppl, 242, pp. 6-14.

Punnett, L., \& Wegman, DH. (2004). Work-related musculoskeletal disorders: the epidemiologic evidence and the debate. J Electromyogr Kinesiol, 14, pp. 13-23.

Reichling, DB., \& Levine, JD. (2009). Critical role of nociceptor plasticity in chronic pain. Trends Neurosci, 32, pp. 611-618.

Ren, K., \& Dubner, R. (2002). Descending modulation in persistent pain: an update. Pain, 100, pp. 1-6.

Richardson, JD., Kilo, S., \& Hargreaves, KM. (1998). Cannabinoids reduce hyperalgesia and inflammation via interaction with peripheral CB1 receptors. Pain, 75, pp. 111-119.

Riedel, W., \& Neeck, G. (2001). Nociception, pain, and antinociception: current concepts. Z Rheumatol, 60, pp. 404-415.

Robergs, RA., Ghiasvand, F., \& Parker, D. (2004). Biochemistry of exercise-induced metabolic acidosis. American Journal of Physiology Regulatory, Integrative and Comparative Physiology, 287, pp. R502-R516.

Robinson, DA., \& Zhuo, M. (2002). Genetic analysis of pain mechanisms. Crit Rev Eukaryot Gene Expr, 12, pp. 275-295.

Rosendal, L., Blangsted, AK., Kristiansen, J., Sogaard, K., Langberg, H., Sjogaard, G., \& Kjaer, M. (2004a). Interstitial muscle lactate, pyruvate and potassium dynamics in the trapezius muscle during repetitive low-force arm movements, measured with microdialysis. Acta physiologica Scandinavica, 182, pp. 379-388.

Rosendal, L., Kristiansen, J., Gerdle, B., Sogaard, K., Peolsson, M., Kjaer, M., Sorensen, J., \& Larsson, B. (2005). Increased levels of interstitial potassium but normal levels of muscle IL-6 and LDH in patients with trapezius myalgia. Pain, 119, pp. 201-209.

Rosendal, L., Larsson, B., Kristiansen, J., Peolsson, M., Sogaard, K., Kjaer, M., Sorensen, J., \& Gerdle, B. (2004b). Increase in muscle nociceptive substances and anaerobic metabolism in patients with trapezius myalgia: microdialysis in rest and during exercise. Pain, 112, pp. 324-334.

Saria, A., Javorsky, F., Humpel, C., \& Gamset, R. (1990). 5-HT3, receptor antagonists inhibit sensory neuropeptide release from the rat spinal cord. Neuroreport, 1, pp. 104-106.

SBU. Metoder för behandling av långvarig smärta. En systematisk litteraturöversikt. Stockholm: Statens beredning för medicinsk utvärdering. 2006.

Schaible, HG., Richter, F., Ebersberger, A., Boettger, MK., Vanegas, H., Natura, G., Vazquez, E., \& Segond von Banchet, G. (2009). Joint pain. Exp Brain Res, 196, pp. 153-162.

Schmelz, M., Schmidt, R., Weidner, C., Hilliges, M., Torebjork, HE., \& Handwerker, HO. (2003). Chemical response pattern of different classes of C-nociceptors to pruritogens and algogens. J Neurophysiol, 89, pp. 2441-2448. 
Schmidt-Wilcke, T. (2008). Variations in brain volume and regional morphology associated with chronic pain. Curr Rheumatol Rep, 10, pp. 467-474.

Schneider, GM., Smith, AD., Hooper, A., Stratford, P., Schneider, KJ., Westaway, MD., Frizzell, B., \& Olson, L. (2010). Minimizing the source of nociception and its concurrent effect on sensory hypersensitivity: An exploratory study in chronic whiplash patients. BMC Musculoskelet Disord, 11, pp. 29.

Schweinhardt, P., \& Bushnell, MC. (2010). Pain imaging in health and disease--how far have we come? J Clin Invest, 120, pp. 3788-3797.

Shah, JP., Danoff, JV., Desai, MJ., Parikh, S., Nakamura, LY., Phillips, TM., \& Gerber, LH. (2008). Biochemicals associated with pain and inflammation are elevated in sites near to and remote from active myofascial trigger points. Arch Phys Med Rehabil, 89, pp. 16-23.

Shah, JP., Phillips, TM., Danoff, JV., \& Gerber, LH. (2005). An in vivo microanalytical technique for measuring the local biochemical milieu of human skeletal muscle. Journal of applied physiology, 99, pp. 1977-1984.

Sjogaard, G., Rosendal, L., Kristiansen, J., Blangsted, AK., Skotte, J., Larsson, B., Gerdle, B., Saltin, B., \& Sogaard, K. (2010). Muscle oxygenation and glycolysis in females with trapezius myalgia during stress and repetitive work using microdialysis and NIRS. Eur J Appl Physiol, 108, pp. 657-669.

Sofat, N., Ejindu, V., \& Kiely, P. (2011). What makes osteoarthritis painful? The evidence for local and central pain processing. Rheumatology (Oxford), pp.

Sommer, C. (2004). Serotonin in pain and analgesia. Mol Neurobiol, 30, pp. 117-125.

Sommer, C. (2006). Is serotonin hyperalgesic or analgesic? Curr Pain Headache Rep, 10, pp. 101-106.

Sommer, C., \& Kress, M. (2004). Recent findings on how proinflammatory cytokines cause pain: peripheral mechanisms in inflammatory and neuropathic hyperalgesia. Neuroscience Letters, 361, pp. 184-187.

Staud, R. (2010). Is it all central sensitization? Role of peripheral tissue nociception in chronic musculoskeletal pain. Curr Rheumatol Rep, 12, pp. 448-454.

Staud, R., Nagel, S., Robinson, ME., \& Price, DD. (2009). Enhanced central pain processing of fibromyalgia patients is maintained by muscle afferent input: a randomized, double-blind, placebo-controlled study. Pain, 145, pp. 96-104.

Stewart, CE., \& Rittweger, J. (2006). Adaptive processes in skeletal muscle: molecular regulators and genetic influences. J Musculoskelet Neuronal Interact, 6, pp. 73-86.

Sullivan, M., Thorn, B., Haythornthwaite, J., Keefe, FJ., Martin, M., Bradley, L., \& Lefebvre, J. (2001). Theoretical perspectives on the relation between catastrophizing and pain. Clin J Pain, 17, pp. 52-64.

Suzuki, R., Rygh, LJ., \& Dickenson, AH. (2004). Bad news from the brain: descending 5-HT pathways that control spinal pain processing. Trends Pharmacol Sci, 25, pp. 613-617.

Svensson, P., Cairns, BE., Wang, K., Hu, JW., Graven-Nielsen, T., Arendt-Nielsen, L., \& Sessle, BJ. (2003). Glutamate-evoked pain and mechanical allodynia in the human masseter muscle. Pain, 101, pp. 221-227.

Svensson, P., Wang, K., Arendt-Nielsen, L., Cairns, BE., \& Sessle, BJ. (2005). Pain effects of glutamate injections into human jaw or neck muscles. J Orofac Pain, 19, pp. 109-118.

Taiwo, YO., \& Levine, JD. (1992). Serotonin is a directly-acting hyperalgesic agent in the rat. Neuroscience, 48, pp. 485-490. 
Tegeder, L., Zimmermann, J., Meller, S., \& Geisslinger, G. (2002 ). Release of algesic substances in human experimental muscle pain. Inflamm Res, 51, pp. 393-402.

Uceyler, N., Schafers, M., \& Sommer, C. (2009). Mode of action of cytokines on nociceptive neurons. Exp Brain Res, 196, pp. 67-78.

Ungerstedt, U. (1991). Microdialysis--principles and applications for studies in animals and man. Journal of internal medicine, 230, pp. 365-373.

Waelgaard, L., Pharo, A., Tonnessen, TI., \& Mollnes, TE. (2006). Microdialysis for monitoring inflammation: efficient recovery of cytokines and anaphylotoxins provided optimal catheter pore size and fluid velocity conditions. Scand J Immunol, 64, pp. 345-352.

Walker, JM., \& Huang, SM. (2002). Endocannabinoids in pain modulation. Prostaglandins Leukot Essent Fatty Acids, 66, pp. 235-242.

Wallin, M., Liedberg, G., Börsbo, B., \& Gerdle, B. (2011). Thermal detection and pain thresholds but not pressure pain thresholds are correlated with psychological factors in women with chronic whiplash associated pain. Clin J Pain, Jul 11. [Epub ahead of print], pp.

Wang, H., Ehnert, C., Brenner, GJ., \& Woolf, CJ. (2006). Bradykinin and peripheral sensitization. Biol Chem, 387, pp. 11-14.

Varney, MA., \& Gereau, RW. (2002 ). Metabotropic glutamate receptor involvement in models of acute and persistent pain: prospects for the development of novel analgesics. Curr Drug Targets CNS Neurol Disord, 1, pp. 283-296.

Weibel, ER., \& Hoppeler, H. (2005). Exercise-induced maximal metabolic rate scales with muscle aerobic capacity. J Exp Biol, 208, pp. 1635-1644.

Wilder-Smith, OH., Tassonyi, E., \& Arendt-Nielsen, L. (2002). Preoperative back pain is associated with diverse manifestations of central neuroplasticity. Pain, 97, pp. 189194.

Woolf, CJ. (2011). Central sensitization: implications for the diagnosis and treatment of pain. Pain, 152, pp. S2-15.

Woolf, CJ., \& Ma, Q. (2007). Nociceptors--noxious stimulus detectors. Neuron, 55, pp. 353364.

Woolf, CJ., \& Salter, MW. (2000). Neuronal Plasticity: increasing the gain in pain. Science, 288, pp. 1765-1768.

Zubakova, R., Gille, A., Faussner, A., \& Hilgenfeldt, U. (2008). Ca2+ signalling of kinins in cells expressing rat, mouse, and human $\mathrm{B} 1 / \mathrm{b} 2$ receptor. Intern Immunopharmacology, 8, pp. 276-281. 


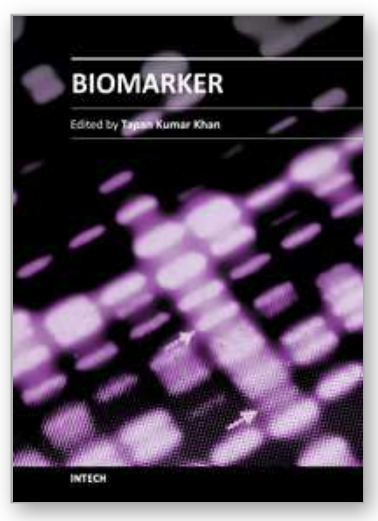

\author{
Biomarker \\ Edited by Prof. Tapan Khan
}

ISBN 978-953-51-0577-0

Hard cover, 392 pages

Publisher InTech

Published online 27, April, 2012

Published in print edition April, 2012

Clinicians, scientists, and health care professionals use biomarkers or biological markers as a measure of a person's present health condition or response to interventions. An ideal -biomarker should have the following criteria: (I) ability to detect fundamental features of the disease, (II) ability to differentiate from other closely related diseases, (III) ability to detect early stages and stages of progression, (IV) the method should be highly reliable, easy to perform and inexpensive, and (V) sample sources should be easily accessible from body. Most of the chapters in this book follow the basic principle of biomarkers.

\title{
How to reference
}

In order to correctly reference this scholarly work, feel free to copy and paste the following:

Björn Gerdle and Britt Larsson (2012). Potential Muscle Biomarkers of Chronic Myalgia in Humans - A Systematic Review of Microdialysis Studies, Biomarker, Prof. Tapan Khan (Ed.), ISBN: 978-953-51-0577-0, InTech, Available from: http://www.intechopen.com/books/biomarker/muscle-biomarkers-of-chronic-myalgia-inhumans-a-systematic-review-of-microdialysis-studies

\section{INTECH}

open science / open minds

\section{InTech Europe}

University Campus STeP Ri

Slavka Krautzeka 83/A

51000 Rijeka, Croatia

Phone: +385 (51) 770447

Fax: +385 (51) 686166

www.intechopen.com

\section{InTech China}

Unit 405, Office Block, Hotel Equatorial Shanghai

No.65, Yan An Road (West), Shanghai, 200040, China 中国上海市延安西路65号上海国际贵都大饭店办公楼 405 单元

Phone: +86-21-62489820

Fax: +86-21-62489821 
(C) 2012 The Author(s). Licensee IntechOpen. This is an open access article distributed under the terms of the Creative Commons Attribution 3.0 License, which permits unrestricted use, distribution, and reproduction in any medium, provided the original work is properly cited. 